\title{
ALGEBRAIC DEFORMATION THEORY
}

\author{
W. STEPHEN PIPER
}

\section{Introduction}

Deformation theory dates back at least to Riemann's 1857 memoir on abelian functions in which he studied manifolds of complex dimension one and calculated the number of parameters (called moduli) upon which a deformation depends. Max Noether in his 1888 paper on the moduli of algebraic surfaces was apparently the first to consider deformations of manifolds of higher dimension. The modern theory of deformations of structures on manifolds was developed extensively in papers by Frölicher-Nijenhuis [2], Kodaira-Spencer [14], [15], KodairaNirenberg-Spencer [13], and Spencer [22], [23]. The study of deformations of algebraic structures was initiated by Gerstenhaber, who, remarking that his methods extend to equationally defined algebraic structures, devoted his work [5] to consideration of associative algebras and graded and filtered rings.

Having the concept of deformation of algebraic structures (principally, associative algebras) and of analytic structures (principally, complex analytic manifolds), we are led to seek a deformation theory of mathematical structures in general. The present paper provides a step towards the development of a generalized deformation theory by introducing a type of cohomology, which we call "deformation cohomology," in the deformation theory of algebraic structures. The deformation cohomology is an algebraic analogue of the cohomology introduced by Haefliger [10] in the deformation theory of structures on manifolds. The latter is developed in greater detail in an unpublished communication from A. Douady to D. C. Spencer. The relationship between the deformation cohomology and the Hochschild cohomology, the latter reflecting the infinitesimal structure, is expressed by an exact, commutative diagram (see $\S \S 9-11)$.

A reasonable deformation theory for mathematical objects should incorporate the notion of a "deformation cohomology" which in turn is related to a cohomology reflecting the infinitesimal structure - the latter cohomology will be called, for simplicity, "infinitesimal cohomology."

Communicated by D.C. Spencer, January 14, 1967. Research supported by the U.S. Army Research Office, Durham, under Contract DA-31-124-ARO(D)-151 and by the Danforth Foundation. 
Our construction of a deformation cohomology in the algebraic theory shows that the principal features of the analytic and algebraic deformation theories can be subsumed under a general treatment, exhibiting in compact form the obstructions to deformations. Throughout we shall exploit the similarity between algebraic deformation theory and the analytic results obtained by Spencer and others.

The principal result of the present paper, contained in $\S 1$, is the definition of an algebraic cohomology theory, $H_{d}^{*}(A, A)$, for an associative algebra $A$ of formal power series in dimensions $0,1,2$, whose second cohomology classes represent equivalence classes of one-parameter families of deformations of $A$, or of $A^{\prime}$, where $A=A^{\prime}[[t]]$. This might be called the cohomology of $A$ with coefficients in the multiplicative group of germs of one-parameter families of deformations of $A$. We shall refer to it more briefly as the (algebraic) deformation cohomology. A similar cohomology is defined for Lie algebras ( $\$ 5)$ and, by means of the deformation cohomologies, the deformation theories of associative and Lie algebras are identified $(\S 6)$. The procedure used in defining the deformation cohomology is valid for any equationally defined algebraic system, and one could just as easily define it for Jordan algebras, Clifford algebras, commutative associative algebras, nilpotent algebras with fixed index of nilpotence, etc. For convenience we cast our discussion throughout in terms of associative algebras.

The basic elements of algebraic deformation theory, previously introduced by Gerstenhaber, are now obtained ( $\$ \S 2-4)$ from the cocycle condition in the deformation cohomology which is, of course, the associativity condition on bilinear maps. Because the original definitions of the deformation cochains and the coboundary operator in dimension 0 were formulated with the intention of demonstrating the identity of the associative and Lie theories, it is necessary to recast these definitions when $A^{\prime}$ is given over a field $k$ with positive characteristic. This is done in $\S 7$, where the equivalence of the two definitions also is shown. Infinitesimal deformations are seen to be Hochschild second cohomology classes of $A^{\prime}$ with coefficients in $A^{\prime}$, and one has a mapping $\rho$ of the deformation cohomology into the Hochschild cohomology. Our interest focuses on determining whether $\rho$ is surjective. One asks under what circumstances a Hochschild two-cocycle can be "integrated" to a deformation. There are obstructions to such an integration, at least formally, but up to now no specific examples have been examined in detail. An obstruction is a Hochschild third cohomology class, and in general, there are an infinite number of obstructions. One conjectures that there are only finitely many obstructions, which is to say that having integrated a cocycle to sufficiently high order, we can determine an extension so that all further obstructions are zero.

The final analogy between the analytic and algebraic theories is the introduction and discussion in $\S 10$ of an exact commutative diagram, which links the deformation and Hochschild cohomologies, in analogy with the analytic case of Spencer [21]. 
Some special aspects of obstructions are discussed in Part II, and our main result is that there are only finitely many obstructions to the extension of a cocycle in $Z^{*}(A, M)$ to $Z^{*}(A, M)[[t]]$ with respect to a given deformation of $A$. Deformations of the module structure of $M$ over $A$ are introduced in $\S 14$. Following one of the philosophical suggestions for a general deformation theory, we induce an infinitesimal cohomology theory whose cochains are multilinear maps of $T(A) \otimes M$ into $A$. The second part concludes with an investigation of relations between deformations of $A$ and of $M$ over $A$, and of extensions of classes in $H^{2}(A, M)$.

I wish to acknowledge my indebtedness to Dr. D. C. Spencer for his assistance and encouragement during the research for and the writing of this paper. Sincere appreciation is also due to Dr. H. Samelson for his comments on the manuscript.

\section{PART I}

\section{ASSOCIATIVE ALGEBRAS}

\section{Notation}

Let $A^{\prime}$ be a given associative algebra with underlying vector space $V^{\prime}$, defined over a field $k$. It is not necessary now to restrict attention to algebras which are finite dimensional over $k$, although this restriction will be useful later. The multiplication of $A^{\prime}$, denoted by $\alpha$, is most often indicated simply by juxtaposition. We currently assume that the characteristic of $k$ is zero, as the positive case is handled separately. Reference to an identity element of $A^{\prime}$ automatically implies that the statement is intended only for a class of unitary algebras. The customary Hochschild [12] cohomology of $A^{\prime}$ with values in itself is denoted by $H^{*}\left(A^{\prime}, A^{\prime}\right)$, and the tensor algebra of $A$ is designated by $T(A)$.

The theory is developed by considering the associative algebra $A=$ $A^{\prime} \otimes_{k} k[[t]]$ of formal power series over $A^{\prime}$, which has as underlying vector space $V=V^{\prime} \otimes_{k} k[[t]]$, where $k[[t]]$ is the ring of formal power series over $k$. We also shall use $\alpha$ to indicate the multiplication on $A$, canonically induced from $A^{\prime}$, and 1 to designate the identity map of $A$, as well as the unit of $A^{\prime}$. The double usages should not cause confusion.

A $p$-linear (over $k$ ) map of $A^{\prime}$ into $A^{\prime}$ induces a $p$-linear (over $k[[t]]$ ) map of $A$ into $A$, as is seen by considering the definition of $A$. Now a $p$ linear map of $A$ into $A$ is said to be "defined from $A^{\prime}$," if it is canonically induced by a $p$-linear map from $A^{\prime}$ to $A^{\prime}$. In the sequel we only shall be concerned with such maps which may be said to be admissible. As we shall not deviate from this convention, it is unnecessary to mention it each time $p$-linear maps of $A$ into $A$ are considered. Also, we shall not distinguish between multilinear maps from $A^{\prime}$ to $A^{\prime}$ and the induced mappings of $A$ into $A$. 
Throughout the first few sections a parallel will be developed between the theory for associative and Lie algebras, culminating in a proof of their coincidence. We shall deal with a Lie algebra $L^{\prime}$, defined over $k$ with underlying vector space $V^{\prime}$ and Lie multiplication $l_{0}$, denoted as usual by [,]. $H^{*}\left(L^{\prime}, L^{\prime}\right)$ is the customary Chevalley-Eilenberg [1] cohomology of $L^{\prime}$, with values in the universal enveloping associative algebra $U\left(L^{\prime}\right)$ of $L^{\prime}$. We also consider $L=L^{\prime} \otimes_{k} k[[t]]$ with $V=V^{\prime} \otimes_{k} k[[t]]$ as underlying vector space.

\section{The deformation cohomology}

Consider an associative algebra $A^{\prime}$ and the extension $A$ of $A^{\prime}$ over the ring of formal power series $k[[t]]$. Define the deformation $q$-cochains, denoted $C_{d}^{q}(A, A)$, of $A$ with values in $A$, for $q=0,1,2$, as follows:

$C_{d}^{0}(A, A)=A$,

$C_{d}^{1}(A, A)=$ the group of vector space automorphisms of $A$ of the form $\Phi_{t}(a)=\sum_{i \geqq 0} \varphi_{i}(a) t^{i}$, where $\varphi_{0}$ is the identity map and the $\varphi_{i}$ are defined from $A^{\prime}$,

$C_{d}^{2}(A, A)=$ the maps of $A \otimes A$ into $A$ of the form $F_{t}(a, b)=\sum_{i \geqq 0} f_{i}(a, b) t^{i}$, where $f_{0}(a, b)=\alpha(a, b)=a b$, and the $f_{i}$ are defined from $A^{\prime}$.

A differential operator $D$ is defined on the $q$-cochains, $q=0,1,2$. For $x \in C_{d}^{0}(A, A), D x$ is the vector space automorphism of $A$ given by

$$
\begin{aligned}
D x(a) & =\exp (x t) \cdot a \cdot \exp (-x t) \\
& =a+(x a-a x) t+\left(\frac{1}{2} x^{2} a-x a x+\frac{1}{2} a x^{2}\right) t^{2}+\cdots
\end{aligned}
$$

For $\Phi_{t} \in C_{d}^{1}(A, A)$

$$
D \Phi_{t}(a, b)=\Phi^{-1}\left(\Phi_{a} \cdot \Phi_{b}\right)
$$

where we abbreviate the notation, writing $\Phi_{a}$ for $\Phi_{t}(a)$. For $F_{t}(a, b) \in$ $C_{d}^{2}(A, A)$

$$
D F_{t}(a, b, c)=F_{t}\left(F_{t}(a, b), c\right)-F_{t}\left(a, F_{t}(b, c)\right)
$$

Remarks. 1) The unit or distinguished element in the set $C_{d}^{2}(A, A)$ is $\alpha$, the original multiplication of $A$. The unit of the group $C_{d}^{1}(A, A)$ is the identity map.

2) $D \Phi_{t}=0$ (or equivalently, $D \Phi_{t}(a, b)=a b$ ) holds if and only if $\Phi_{t}$ is an algebra automorphism. 0 .

3) $F_{t}(a, b)$ is an associative multiplication on $A$ if and only if $D F_{t}=$ 
4) Everything indicated in this section and the next can be given in precisely the same fashion, except for $D$ on the 0 -cochains, when the characteristic of the ground field is not zero. Even here the result is equivalent (cf. §7). However, the later discussions for Lie algebras would not be valid.

Proposition 1. D is a well-defined differential operator, that is,

$$
\text { 1) } \quad D^{2} x(a, b)=a b, \quad \text { 2) } \quad D^{2} \Phi_{t}(a, b, c)=0 .
$$

The proof is an immediate consequence of the definitions.

The cohomology of this differential complex, called the (algebraic) deformation cohomology, is denoted $H_{d}^{q}(A, A), q=0,1,2$. It is not defined in higher dimensions, nor, analogously, was deformation cohomology defined for $q \geqq 2$ in the analytic development. Since $H_{d}^{0}(A, A)$ is the center of $A$, it is a commutative algebra; $H_{d}^{1}(a, A)$ is a group of (equivalence classes of) algebra automorphisms; and $H_{d}^{2}(A, A)$ is a cohomology set with distinguished element, which is the class of $\alpha$.

\section{Connection with deformation theory}

The cochain $\Phi_{t}(a) \in C_{d}^{1}(A, A)$ is called a germ of (vector space) automorphism of $A^{\prime}$ or a one-parameter family of (vector space) automorphisms, and $Z_{d}^{1}(A, A)$ is the subgroup of algebra automorphisms of $A$. An algebra automorphism $\Phi_{t}(a)$ is said to be trivial, or equivalent to the identity, if it is an inner automorphism of $A$. As a consequence of the definitions, we have

Proposition 2. The equivalence classes of germs of automorphisms of the algebra $A^{\prime}$ are in natural one-one correspondence with the cohomology classes $H_{d}^{1}(A, A)$.

More important for our considerations is the following:

Proposition 3. The equivalence classes of deformations of the algebra $A^{\prime}$ are in natural one-one correspondence with the cohomology classes $H_{d}^{2}(A, A)$.

A deformation of the algebra $A^{\prime}[5$, p. 62] is given by an element of $Z_{d}^{2}(A, A)$, and two deformations $F_{t}, G_{t}$ are said to be equivalent if there is a (vector space) automorphism $\Phi_{t}(a)=a+\sum_{i \geqq 1} \varphi_{i}(a) t^{i}$ of $V$ such that $F_{t}(\dot{a}, b)=\Phi^{-1}\left(G_{t}\left(\Phi_{a}, \Phi_{b}\right)\right)$. But this is to say that $F_{t}, G_{t}$ considered as elements of $Z_{d}^{2}(A, A)$ are cohomologous.

We now proceed to prove that differentiation with respect to $t$ at $t=$ 0 maps the deformation $q$-cochains, $q=0,1,2$, into the Hochschild $q$ cochains and, moreover, this mapping takes $H_{d}^{q}(A, A)$ into $H^{q}\left(A^{\prime}, A^{\prime}\right)$. In general the mapping is neither injective nor surjective.

For $x \in H_{d}^{0}(A, A)=Z_{d}^{0}(A, A), D x$ must be the identity automorphism of $A$, i.e., $\exp (t x) \cdot a \cdot \exp (-t x)=a$. But this is true if and only if $x$ lies in the center of $A$, or equivalently, $x \in Z^{0}(A, A)$. Writing $x=\sum_{i \geqq 0} v_{i} t^{i}, x \in Z^{0}(A, A)$ implies $\left.\frac{d x}{d t}\right|_{t=0}=v_{1} \in Z^{0}\left(A^{\prime}, A^{\prime}\right)$. 
Differentiating $\Phi_{t}=1+\sum_{i \geqq 1} \varphi_{i} t^{i}$ at $t=0$, we obtain the infinitesimal automorphism $\varphi_{1}$. That $\Phi_{t}$ be a cocycle in $C_{d}^{1}(A, A)$ implies certain restrictions on the $\varphi_{i}$. Namely, for all nonnegative $r$,

$$
\varphi_{r}(a, b)=\sum_{\substack{p+q=r \\ p, q \geqq 0}} \varphi_{p}(a) \varphi_{q}(b)
$$

or equivalently, making use of the Hochschild coboundary operator $\delta$ and introducing the cup product of Hochschild 1-cochains $\varphi \cup \phi(a, b)=$ $\varphi(a) \phi(b)$, we may write (1) as

$$
\begin{aligned}
& \delta \varphi_{1}=0, \\
& \delta \varphi_{r}=-\sum_{\substack{p+q=r \\
p, q>0}} \varphi_{p} \cup \varphi_{q}, \quad r \geqq 2 .
\end{aligned}
$$

Proposition 4. Cohomologous algebra automorphisms have Hochschild cohomologous infinitesimal automorphisms.

Automorphisms $\Phi_{t}, \Psi_{t}$ are cohomologous if there is an $x \in C_{d}^{0}(A, A)$ such that

$$
\Psi_{t}^{-1}\left(\Phi_{t}(a)\right)=D x(a)=\exp (t x) \cdot a \cdot \exp (-t x)
$$

or equivalently, for $\Psi \in Z_{d}^{1}(A, A)$,

$$
\begin{aligned}
\Phi_{t}(a) & =\Psi_{t}(\exp (t x) \cdot a \cdot \exp (-t x)) \\
& =\exp \left(t \Psi_{x}\right) \cdot \Psi_{a} \cdot \exp \left(-t \Psi_{x}\right)
\end{aligned}
$$

or, hence,

$$
\Phi_{t}(a)=D \Psi_{x}\left(\Psi_{a}\right)
$$

It is notationally simpler to allow $\chi$ to stand for $\exp (t x)$. Thus, $\Phi_{t}$ and $\Psi_{t}$ are said to be equivalent if there is a $\chi(=\exp (t x)) \in C_{d}^{0}(A, A)=$ $A$ such that

$$
\Psi_{t}^{-1}\left(\Phi_{t}(a)\right)=\chi a \chi^{-1}=D x(a)
$$

This has the further, and significant, advantage of allowing the formulas and the theory to hold in the case characteristic $k \neq 0$, which case is treated in $\S 7$.

Proof of Proposition 4. If $\Psi_{t}(a)=a+\sum_{i \geqq 1} \phi_{i}(a) t^{i}$, then $\Psi_{t}^{-1}(\chi)=$ $\chi^{-1}-\chi^{-1} \phi_{1}(\chi) \chi^{-1} t+\cdots$. Expressing $\Phi_{t}(a)=\Psi_{t}\left(\chi a \chi^{-1}\right)=\Psi_{t}(\chi) \Psi_{t}(a) \Psi_{t}^{-1}(\chi)$ in powers of $t$, we have 
(3)

$$
\Phi_{t}(a)=\chi a \chi^{-1}+\left(\phi_{1}(\chi) a \chi^{-1}+\chi \phi_{1}(a) \chi^{-1}-\chi a \chi^{-1} \phi_{1}(\chi) \chi^{-1}\right) t+\cdots .
$$

Recalling $\chi=\exp (t x)$, we see that (3) becomes

$$
\begin{aligned}
\Phi_{t}(a) & =a+\left(x a-a x+\phi_{1}(a)\right) t+\cdots \\
& =a+\left(\phi_{1}(a)+\delta y(a)\right) t+\cdots,
\end{aligned}
$$

where $y$ is the Hochschild 0 -cochain $-x$, which completes the proof. Therefore, differentiation with respect to $t$ at $t=0$ induces a map

$$
\rho: H_{d}^{1}(A, A) \rightarrow H^{1}\left(A^{\prime}, A^{\prime}\right)
$$

Note that in the special case when $A^{\prime}$ is a finite dimensional algebra over $k$, with char $k=0, \rho$ is onto, since for given $\varphi_{1} \in Z^{1}\left(A^{\prime}, A^{\prime}\right), \Phi_{t}=$ $\exp \left(\varphi_{1} t\right)$ lies in $Z_{d}^{1}(A, A)$ (see $\left.\S 4\right)$.

Proposition 5. Given $\Phi_{t}=1+\varphi_{n} t^{n}+\cdots$ in $Z_{d}^{1}(A, A)$, then $\varphi_{n} \in$ $Z^{1}\left(A^{\prime}, A^{\prime}\right)$, and if $\varphi_{n}$ is cohomologous to zero, $\Phi_{t}$ is equivalent to a oneparameter family of algebra automorphisms $\Psi_{t}=1+\varphi_{n+1} t^{n+1}+\cdots$.

Proof. From (2) $\varphi_{1}=\cdots=\varphi_{n-1}=0$ implies that $\delta \varphi_{n}=0$, and hence $\varphi_{n} \in Z^{1}\left(A^{\prime}, A^{\prime}\right)$. If $\varphi_{n} \in B^{1}\left(A^{\prime}, A^{\prime}\right)$, there is a $y \in A^{\prime}$ such that $\varphi_{n}(a)=\delta y(a)=a y-y a$.

Let $x \in C_{d}^{0}(A, A)=A$ be given by $x=y t^{n-1}$ and set $\chi=\exp (x t)=$ $1+y t^{n}+\cdots$. Then $\Phi_{t}(a)$ is cohomologous, by definition, to $\Phi_{t}\left(\chi a \chi^{-1}\right)$, and

$$
\begin{aligned}
\Phi_{t}\left(\chi a \chi^{-1}\right) & =\left(1+y t^{n}\right) a\left(1-y t^{n}\right)+\varphi_{n}(a) t^{n}+\cdots \\
& =a+o\left(t^{n+1}\right)
\end{aligned}
$$

completing the proof.

Corollary. $H^{1}\left(A^{\prime}, A^{\prime}\right)=0$ implies that all automorphisms of $A$ (or of $A^{\prime}$ ) are inner automorphisms.

A cocycle $\varphi$ in $Z^{1}\left(A^{\prime}, A^{\prime}\right)$ is integrable if it lies in the image of $\rho$. The integrability question is a function of the cohomology class. More explicitly, we have

Proposition 6. Suppose $\varphi \in Z^{1}\left(A^{\prime}, A^{\prime}\right)$ is integrable. Then $\varphi$ is integrable whenever $\phi$ is cohomologous to $\varphi$.

Proof. Let $\phi(a)=\varphi(a)+\delta \beta(a)=\varphi(a)+a \beta-\beta a$, for some $\beta \epsilon$ $C^{0}(A, A)=A$. Consider $\Psi_{t}(a)=\Phi_{t}\left(\chi^{-1} a \chi\right)$ where $\chi=\exp (t \beta)$, and $\Phi_{t} \in \rho^{-1}(\varphi)$, i.e., $\Phi_{t}(a)$ has $\varphi(a)$ as its infinitesimal automorphism.

$$
\begin{aligned}
\Psi_{t}(a) & =\chi^{-1} a \chi+\varphi\left(\chi^{-1} a \chi\right)+\cdots \\
& =a+(-\beta a+a \beta) t+\varphi(a) t+\cdots=a+\phi(a) t+\cdots
\end{aligned}
$$

That is, $\phi$ is the infinitesimal automorphism of $\Psi_{t}$ and is integrable. 
The requirement that $D F_{t}(a, b, c)=F_{t}\left(F_{t}(a, b), c\right)-F_{t}\left(a, F_{t}(b, c)\right)=$ 0 , for $F_{t}=\alpha+\sum_{i \geqq 1} f_{i} t^{i}$, expressed in powers of $t$, implies that the $f_{i}$ satisfy for all nonnegative $r$ :

$$
\sum_{\substack{p+q=r \\ p, q \geqq 0}} f_{p}\left(f_{q}(a, b), c\right)-f_{p}\left(a, f_{q}(b, c)\right)=0,
$$

or equivalently, for positive $r$,

$$
\begin{gathered}
\delta f_{1}(a, b, c)=0, \\
\delta f_{r}=\sum_{\substack{p+q=r \\
p, q>0}} f_{p} * f_{q}=\frac{1}{2} \sum_{\substack{p+q=r \\
p, q>0}}\left[f_{p}, f_{q}\right],
\end{gathered}
$$

where $f_{p} * f_{q}(a, b, c)=f_{p}\left(f_{q}(a, b), c\right)-f_{p}\left(a, f_{q}(b, c)\right)$ and $\left[f_{p}, f_{q}\right]=f_{p} *$ $f_{q}+f_{q} * f_{p}$. Differentiating $F_{t}$ at $t=0$, we obtain the infinitesimal deformation $f_{1}$, which by (5) is a cocycle in $C^{2}\left(A^{\prime}, A^{\prime}\right)$.

Proposition 7. If $F_{t}, G_{t}$ are cohomologous multiplications, then $f_{1}, g_{1}$ are cohomologous Hochschild cocycles.

Proof. $F_{t} \sim G_{t}$ implies there exists a 1-cochain $\Phi_{t} \in C_{d}^{1}(A, A)$ such that $F_{t}(a, b)=\Phi^{-1}\left(G_{t}\left(\Phi_{a}, \Phi_{b}\right)\right)$, and thus

$$
\begin{aligned}
F_{t}(a, b) & =G_{t}\left(\Phi_{a}, \Phi_{b}\right)-\varphi_{1}\left(G_{t}\left(\Phi_{a}, \Phi_{b}\right)\right) t+\cdots \\
& =\Phi_{a} \cdot \Phi_{b}+g_{1}\left(\Phi_{a}, \Phi_{b}\right) t-\varphi_{1}\left(\Phi_{a} \cdot \Phi_{b}\right) t+\cdots \\
& =a b+\left(a \varphi_{1}(b)+\varphi_{1}(a) b\right) t+g_{1}(a, b) t-\varphi_{1}(a b) t+\cdots \\
& =a b+\left(g_{1}(a, b)+\delta \varphi_{1}(a, b)\right) t+\cdots
\end{aligned}
$$

Therefore, $f_{1}$ and $g_{1}$ represent the same cohomology class in $H^{2}\left(A^{\prime}, A^{\prime}\right)$, and we have that each cohomology class in $H_{d}^{2}(A, A)$ determines a class in $H^{2}\left(A^{\prime}, A^{\prime}\right)$. Denote this mapping by $\rho=\left.\frac{\partial}{\partial t}\right|_{t=0}$.

Proposition 8. Given nontrivial $F_{t} \in Z_{d}^{2}(A, A), F_{t}$ is equivalent to a one-parameter family of deformations $G_{t}=\alpha+g_{n} t^{n}+g_{n+1} t^{n+1}+$ $\cdots$, where the first nonvanishing cochain $g_{n}$ is in $Z^{2}\left(A^{\prime}, A^{\prime}\right)$ and is not cohomologous to zero.

The proof is similar to that of Proposition 5 and is omitted (cf. [5, p. 65]).

Corollary. $H^{2}\left(A^{\prime}, A^{\prime}\right)=0$ implies there are no nontrivial deformations of $A$ ( or of $\left.A^{\prime}\right)$.

Again, a cocycle $f_{1}$ in $Z^{2}\left(A^{\prime}, A^{\prime}\right)$ is integrable if it lies in the image of $\rho$, and we have

Proposition 9. If $f_{1} \in Z^{2}\left(A^{\prime}, A^{\prime}\right)$ is integrable, then so is any cocycle $g_{1}$ cohomologous to $f_{1}$. 


\section{The deformation equation and integrability}

The previous section concluded with a note on integrability, and this concept is developed now more fully.

Given a Hochschild 2-cocycle $f_{1}$, which as we shall see can be regarded as a tangent vector to an algebraic variety, we ask the integrability question. When can $f_{1}$ be "integrated" or extended by elements of $C_{d}^{2}(A, A)$ to a deformation 2-cochain, satisfying an appropriate structure condition, which in terms of the deformation cohomology is the condition that it be an associative algebra? This requirement parallels that implied in discussions of the analytic deformation cohomology in the manifold theory and is also realizable in terms of a deformation equation. The deformation equation can be stated, using the Hochschild coboundary $\delta$, as

$$
\delta v-\frac{1}{2}[v, v]=0
$$

where $v$ has the form $f_{1} t+f_{2} t^{2}+\cdots$.

The equivalence of determining such a $v$ and of having $F_{t}=\alpha+$ $\sum_{i \geqq 1} f_{i} t^{i} \in Z_{d}^{2}(A, A)$ is clear from setting $v=\sum_{i \geqq 1} f_{i} t^{i}$ and using the bracket defined in the preceding section. We have

$$
F_{t} \in Z_{d}^{2}(A, A) \Longleftrightarrow[\alpha+v, \alpha+v]=0,
$$

and

$$
0=[\alpha+v, \alpha+v]=2[v, \alpha]+[v, v]
$$

implies

$$
\delta v=\frac{1}{2}[v, v]=v * v
$$

which is, of course, the same as (6).

A "deformation equation" can be stated also in the case of automorphisms. Expressing $\Phi_{t}=1+\sum_{i \geqq 1} \varphi_{i} t^{i}$ by $1+\omega$, the condition that $\Phi_{t}$ be an (associative) algebra automorphism is that $\Phi_{t}(a b)=\Phi_{t}(a) \Phi_{t}(b)$, i.e., $\Phi_{t} \in Z_{d}^{1}(A, A)$, and thus

$$
\begin{aligned}
a b+\omega(a b) & =(a+\omega(a))(b+\omega(b)) \\
& =a b+a \omega(b)+\omega(a) b+\omega(a) \omega(b),
\end{aligned}
$$

from which $\delta \omega+\omega \cup \omega=0$. 
Given $f_{1} \in Z^{2}\left(A^{\prime}, A^{\prime}\right)$ and $f_{i} \in C^{2}\left(A^{\prime}, A^{\prime}\right), 1<i<n$, satisfying (6) for all $r<n$, we say $f_{1}$ is integrated up to order $n-1$. The integration equation may be stated recursively. Can we find $f_{n} \in C^{2}\left(A^{\prime}, A^{\prime}\right)$ such that

$$
\delta f_{n}=\sum_{\substack{p+q=n \\ p, q>n}} f_{p} * f_{q} ?
$$

This is to ask whether $\omega_{n}=\sum_{\substack{p+q=n \\ p, q>n}} f_{p} * f_{q}$ is cohomologous to zero. The class of $\omega_{n}$ is said to be the $n^{\text {th }}$ obstruction to the integration of $f_{1}$. Obstructions are Hochschild 3-cohomology classes, as proven by Gerstenhaber [5]. Should it be the case that $H^{3}\left(A^{\prime}, A^{\prime}\right)=0$, all obstructions vanish and the formal question of integration is settled in the affirmative. By formal in this context one means that, yes, an $f_{n}$ with the requisite property does exist for each $n$. The cocycle $f_{1}$ is said to be integrated when an appropriate $f_{n}$ has been chosen, for each $n>1$. The primary obstruction (equal to the class of $\omega_{2}=f_{1} * f_{1}$ ) depends only upon the Hochschild class of $f_{1}$, while the higher obstructions $\left\{\omega_{n}\right\}$ depend upon the choice of $f_{2}, \cdots, f_{n-1}$, as well as upon $f_{1}$.

\section{Integration of infinitesimal automorphisms}

The integration question considered in this section asks to what extent is the differentiation map $\rho: H_{d}^{1}(A, A) \rightarrow H^{1}\left(A^{\prime}, A^{\prime}\right)$ onto. That is, given an infinitesimal automorphism $\varphi$ in $Z^{1}\left(A^{\prime}, A^{\prime}\right)$, can we find an associative algebra automorphism $\Phi_{t}=1+\sum_{i \geqq 1} \varphi_{i} t^{i}$ in $Z_{d}^{1}(A, A)$ such that $\varphi_{1}=\varphi$ ? The answer to be proven shortly is easily stated. Yes, $\rho$ is onto when $k$ has characteristic zero. The following proof also holds for algebras with positive characteristic $p$, when $p>2 q$, where the $q^{\text {th }}$ iterate of $\varphi$ is the zero map. The more general case of positive characteristic is not considered here, except to note the existence of actual obstructions for the group algebra of $Z_{p}$.

Given $\varphi \in Z^{1}\left(A^{\prime}, A^{\prime}\right)$, define

$$
\begin{array}{ll}
\varphi_{1}(a) & =\varphi(a), \quad \varphi_{2}(a)=\frac{1}{2} \varphi_{1}\left(\varphi_{1}(a)\right) \\
\varphi_{3}(a) & =\frac{1}{3} \varphi_{1}\left(\varphi_{2}(a)\right), \quad \text { etc. }
\end{array}
$$

Lemma 1. By the associativity of the composition of automorphisms,

$$
\varphi_{i}\left(\varphi_{j}(a)\right)=\left(\begin{array}{c}
i+j \\
i
\end{array}\right) \varphi_{i+j}(a)
$$

The proof is straightforward as one quickly sees that the numerical coefficients involved are simply the binomial coefficients. 
Consider $\Phi_{t}=1+\sum_{i \geqq 1} \varphi_{i} t^{i}$, where the $\varphi_{i}$ are as given above. Then $\Phi_{t}=e^{t \varphi}$.

Proposition 10. $e^{t \varphi}$ belongs to $Z_{d}^{1}(A, A)$, i.e., is an algebra automorphism.

The necessary and sufficient condition that $\Phi_{t}=e^{t \varphi}$ be an algebra automorphism is that

$$
\varphi_{r}(a b)=\sum_{\substack{p+q=r \\ p, q \geqq 0}} \varphi_{p}(a) \varphi_{q}(b) .
$$

The proof proceeds by induction. Observe that (10) is trivial for $r=0$ and holds for $r=1$, as $\varphi_{1}=\varphi \in Z^{1}\left(A^{\prime}, A^{\prime}\right)$. Assume (10) holds for all $r \leqq n-1$. Then,

$$
\begin{aligned}
\varphi_{n}(a b) & =\frac{1}{n} \varphi_{1}\left(\varphi_{n-1}(a b)=\frac{1}{n} \sum_{\substack{r+s=n-1 \\
r, s \geqq 0}} \varphi_{1}\left(\varphi_{r}(a) \varphi_{s}(b)\right)\right. \\
& =\frac{1}{n} \sum_{\substack{r+s=n-1 \\
r, s \geqq 0}} \varphi_{1}\left(\varphi_{r}(a)\right) \varphi_{s}(b)+\varphi_{r}(a) \varphi_{1}\left(\varphi_{s}(b)\right) \\
& =\frac{1}{n} \sum_{\substack{r+s=n-1 \\
r, s \geqq 0}}(r+1) \varphi_{r+1}(a) \varphi_{s}(b)+\frac{1}{n} \sum_{\substack{j=n-1 \\
i, j \geqq 0}}(j+1) \varphi_{i}(a) \varphi_{j+1}(b) \\
& =\sum_{\substack{p+q=n \\
p, q>0}}(p+q) \varphi_{p}(a) \varphi_{q}(b) \\
& \left.=\sum_{\substack{p+a=n \\
p, q>0}} \varphi_{p}(a) \varphi_{q}(b), \varphi_{n}(b)\right)+\frac{1}{n+q}(p) \text { for all } n \geq 0 .
\end{aligned}
$$

q.e.d.

Given $\varphi_{1}, \cdots, \varphi_{n}$ in $C^{1}\left(A^{\prime}, A^{\prime}\right)$ satisfying

$$
\delta \varphi_{r}=\sum_{\substack{p+q=r \\ p, q>0}} \varphi_{p} \cup \varphi_{q}, \quad \text { for } r=1, \cdots, n,
$$

the Hochschild class of $\sum_{\substack{p=n+1 \\ p, q>0}} \varphi_{p} \cup \varphi_{q}$ is called the $(n+1)^{t h}$ obstruction to the integration of $\varphi_{1}$. If and only if this class is the zero coho-

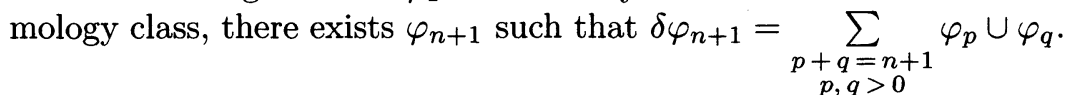

We just have proven that given $\varphi_{1}$ we can pick $\varphi_{i}$ in order to obtain a global algebra automorphism with infinitesimal $\varphi_{1}$. However, given $\varphi_{1}, \cdots, \varphi_{n}$ satisfying (11), there may or may not be a global automorphism which modulo $t^{n+1}$ is equal to $1+\sum_{i=1}^{n} \varphi_{i}$. This is reflected in the fact that the map $\pi_{1}$ of $\S 10$ is not onto.

Consider now the unitary group algebra over $k$ (when characteristic $k=p$ ) of $Z_{p}$. For each prime $p$, a derivation of this algebra can be exhibited which is not integrable (cf. [5, p. 89]). 


\section{The Lie deformation cohomology}

Consider the Lie algebra $L^{\prime}$, and $\mathrm{L}=L^{\prime}[[t]]$. Then define $C_{d}^{0}(L, L)=L$, and $C_{d}^{1}(L, L)$ to be the group of vector space automorphisms of $L$ of the form $\Phi_{t}=\sum_{i \geqq 0} \varphi_{i} t^{i}$, where $\varphi_{0}(a)=a$, and the $\varphi_{i}$ are defined from $L^{\prime}$.

Let $C_{d}^{2}(L, L)$ be the group of bilinear, skew-symmetric maps of $L$ into itself of the form $B_{t}=\sum_{i \geqq 0} B_{i} t^{i}$, where $B_{0}(a, b)=[a, b], B_{1}$ maps into

$L$, the $B_{i}$ map into the universal enveloping associative algebra $U(L)$ of $L$ for $i>1$, and for $i \geqq 1$, the $B_{i}$ are defined from $L^{\prime}$.

For $x \in L, D x \in C_{d}^{1}(L, L)$ is the inner automorphism of $L$ determined by $x$. Explicitly,

$$
D x(y)=\exp (a d x t)(y)=y+[x, y] t+\frac{1}{2}[x,[x, y]] t^{2}+\cdots .
$$

And,

$$
\begin{aligned}
& D \Phi_{t}(a, b)=\Phi^{-1}\left(\left[\Phi_{a}, \Phi_{b}\right]\right), \\
& D B_{t}(a, b, c)=B_{t}\left(B_{t}(a, b), c\right)+B_{t}\left(B_{t}(b, c), a\right)+B_{t}\left(B_{t}(c, a), b\right) .
\end{aligned}
$$

As in $\S 1$ (Proposition 1 ), $D$ is a well-defined differential operator.

Consequently, $D \Phi_{t}=0$ if and only if $\Phi_{t}$ is a Lie algebra automorphism, and $B_{t}$ defines a Lie multiplication (that is, satisfies the Jacobi identity) if and only if $D B_{t}=0$. We also have

Proposition 11. The equivalence classes of deformations of the Lie algebra $L^{\prime}$ are in natural one-one correspondence with the cohomology classes $H_{d}^{2}(L, L)$.

Everything is defined just as in the previous three sections, and the analogues of Propositions 2-9 are immediate.

Remark. For other equationally defined algebras - commutative, Jordan, etc.- - the deformation two-coboundary would be the defining relation, just as in the associative and Lie cases.

This completes the introduction of the algebraic analogue of the analytic cohomology.

\section{Identity of the associative and Lie theories}

Theorem 1. There is a natural isomorphism between the one-parameter automorphisms and deformations of Lie algebras and of their universal enveloping algebras.

That is, to every deformation of a Lie (associative) algebra, one can associate a deformation of an associative (Lie) algebra, and similarly for automorphisms.

Let $L^{\prime}$ be a Lie algebra, $L=L^{\prime}[[t]]$, and $A=U(L)$, the universal enveloping associative algebra of $L$. We define a natural map $\gamma: H_{d}^{q}(L, L) \rightarrow H_{d}^{q}(A, A)$. By definition of $A$ as the universal enveloping associative algebra of $L$, Lie algebra automorphisms $\Phi_{t}$ of 
$L$ extend to unique algebra automorphisms $\gamma\left(\Phi_{t}\right)$ of $A$, and $\gamma$ so defined carries inner automorphisms into inner automorphisms. Therefore, $\gamma: H_{d}^{1}(L, L) \rightarrow H_{d}^{1}(A, A)$. The map on the 2-level is obtained in a similar fashion; $B_{t} \in Z_{d}^{2}(L, L)$ gives a one-parameter family $\left\{L_{t}\right\}$ of Lie algebras deforming $L$, and hence one has a one-parameter family of enveloping associative algebras $\left\{A_{t}\right\}$, which is a deformation of $A$. And $A_{t}=T(L) / R_{t}$, where $R_{t}$ is the ideal of $T(L)$ generated by $B_{t}(a, b)-a \otimes b+b \otimes a$. This set of multiplications on $A$ is defined to be $\gamma\left(B_{t}\right) \in Z_{d}^{2}(A, A)$. Equivalence of deformations is preserved, and consequently, $\gamma: H_{d}^{2}(L, L) \rightarrow H_{d}^{2}(A, A)$.

Theorem 2. The map $\gamma: H_{d}^{q}(L, L) \rightarrow H_{d}^{q}(A, A)$ is injective.

On the 0-level $\gamma$ is an imbedding and, for $q=1,2, \gamma$ takes a cocycle into a unique extension. As two cocycles cannot have the same extension, $\gamma$ is injective.

As $A$ is a filtered algebra with $F_{1}(A)=L$, restricting cocycles representing classes in $H_{d}^{q}(A, A)$ to $F_{1}(A)$, one has a map $H_{d}^{q}(A, A) \rightarrow$ $H_{d}^{q}(L, L), q=0,1,2$. Hence $\gamma$ is onto. This completes the discussion of Theorem 1.

Further, given an associative algebra, one defines a Lie algebra $L(A)=L$ over the underlying vector space $V$ of $A$, by introducing the commutator bracket $[a, b]=a b-b a$. There is an immediate relation between $H_{d}^{q}(A, A)$ and $H_{d}^{q}(L, L)$. Moreover, $\Phi_{t} \in Z_{d}^{1}(A, A)$ is an element of $Z_{d}^{1}(L, L)$, and $\Phi_{t} \in B_{d}^{1}(A, A)$ is an element of $B_{d}^{1}(L, L)$. Given $F_{t} \in Z_{d}^{2}(A, A)$, define $B_{t} \in Z_{d}^{2}(L, L)$ by $B_{t}(a, b)=F_{t}(a, b)-F_{t}(b, a)$. If $F_{t}$ is a coboundary, then so is $B_{t}$. As a result we have a map $H_{d}^{q}(A, A) \rightarrow H_{d}^{q}(L, L)$, and accordingly, every deformation (automorphism) of an associative algebra can be considered a deformation (automorphism) of a Lie algebra.

\section{The case of characteristic $p \neq 0$}

Assume $A^{\prime}$ has an identity element. For char $k \neq 0, C_{d}^{1}(A, A), C_{d}^{2}(A, A)$ and $D^{1}, D^{2}$ are well defined. An alternate and equivalent definition of $C_{d}^{0}(A, A)$, and $D^{0}$ can be given so that these are well defined as well. Intuitively, the 0 -cochains are those elements of $A$ which give rise to inner automorphisms of $\mathrm{A}$, and the coboundary of such an element is this inner automorphism. The definitions of $\S 1$ for 0 -cochains and the coboundary operator $D^{0}$ were formulated with the discussion of the deformation cohomology of Lie algebras in mind. However, the following formulation is independent of char $k$ (and hence is the necessary formulation for char $k \neq 0$ ).

Let $C$ be the set of invertible elements of $A$ whose constant term lies in the center of $A$ (equivalently, of $A^{\prime}$ ). With this definition for 0cochains, the 0 -coboundary operator is conjugation, i.e., the coboundary of $x \in C$ is the inner automorphism of $A$ given by $x a x^{-1}$. 
Lemma 2. When char $k=0$, every inner automorphism of $A$, defined by conjugation by $x \in C \subset A$, is equal to conjugation by an element of $A$, of the form $\exp (t x), x \in A$.

In other words, the lemma says that $C$ and $C_{d}^{0}(A, A)$ are isomorphic and that the two definitions of coboundary operator are equivalent. In each case the coboundary of a 0-cochain is an inner automorphism defined by the 0 -cochain.

We recall that $x \in C$ may be expressed in powers of $t$ by

$$
x=v_{0}+v_{1} t+v_{2} t^{2}+\cdots, \quad v_{i} \in A^{\prime}
$$

where $v_{0}$ lies in the center of $A^{\prime}$ and is invertible. Dividing out $v_{0}$ does not affect the inner automorphism $I_{x}(a)=x a x^{-1}$, and so without loss of generality we may take $v_{0}$ to be 1 , the identity element of $A^{\prime}$.

Then $x^{-1}$ has the form, in powers of $t, x^{-1}=1+\sum_{i \geqq 1} y_{i} t^{i}$, where $y_{1}=-v_{1}$, and in general,

$$
y_{r}=-\sum_{\substack{p+q=r \\ p, q>0}} v_{p} y_{q}-v_{r}
$$

Thus,

$$
\begin{aligned}
x a x^{-1} & =\left(1+v_{1} t+v_{2} t^{2}+\cdots\right) a\left(1-v_{1} t+\left(v_{1}^{2}-v_{2}\right) t^{2}+\cdots\right) \\
& =a+\left(v_{1} a-a v_{1}\right) t+\left(v_{2} a-v_{1} a v_{1}+a v_{1}^{2}-a v_{2}\right) t^{2}+\cdots
\end{aligned}
$$

Substituting in $\exp (t \chi)$ the power series in $t$ of $\chi=\sum_{i \geqq 0} \chi_{i} t^{i}$, we have

$$
\begin{aligned}
\exp (t \chi)=1+\chi_{0} t & +\left(\chi_{1}+\frac{1}{2} \chi_{0}^{2}\right) t^{2} \\
& +\left(\chi_{2}+\frac{1}{2} \chi_{0} \chi_{1}+\frac{1}{2} \chi_{1} \chi_{0}+\frac{1}{6} \chi_{0}^{3}\right) t^{3}+\cdots
\end{aligned}
$$

Therefore,

$$
\begin{aligned}
& \exp (t \chi) a \exp (-t \chi)=a+\left(\chi_{0} a-a \chi_{0}\right) t \\
& \quad+\left(\chi_{1} a+\frac{1}{2} \chi_{0}^{2} a-\chi_{0} a \chi_{0}+\frac{1}{2} a \chi_{0}^{2}-a \chi_{1}\right) t^{2}+\cdots
\end{aligned}
$$

Comparing (13) and (14), one can solve for $\chi$ so that $(13)=(14)$, by letting

$$
\begin{aligned}
& \chi_{0}=v_{1} \\
& \chi_{1}=v_{2}-\frac{1}{2} v_{1}^{2} \\
& \chi_{2}=v_{3}+\frac{1}{3} v_{1}^{3}-\frac{1}{2} v_{1} v_{2}-\frac{1}{2} v_{2} v_{1}, \quad \text { etc. }
\end{aligned}
$$


This establishes the general pattern expressing successive $\chi_{i}$ in terms of the $v_{j}$. But this is to say that conjugation by elements of $C$ is equivalent to conjugation by elements of the form $\exp (t \chi), \chi \in A$, completing the proof of the lemma. The modified differential operator will be denoted by $D^{\prime}$.

The results of $\S 2$ continue to hold for characteristic $k \neq 0$ when the deformation cohomology is reinterpreted as above. No change is required in the statement of the propositions, while the proofs need little or no alteration. For the proof of Proposition 4 , replace $D$ by $D^{\prime}$, and $\chi=\exp (t x)$ by $x=v_{0}+v_{1} t+v_{2} t^{2}+\cdots \in C$. In the proof of Proposition 5, let $x=1+y t$, and for Proposition 6 , let $x=1+\beta t$ replace $\chi$.

A similar modification for Lie algebras can be given only in special cases, as the exponential map is essential in defining the Lie algebra inner automorphism determined by an element of the algebra. However, the results of $\S \S 3$ and 5 pertaining to deformations (i.e., 2-cocycles) are independent of the characteristic, and so continue to hold for positive characteristic different from 2. Nijenhuis-Richardson [20] have devoted several sections to discussion of the case char $k=2$.

\section{Deformation theory in terms of structure constants}

The associativity of an algebra $A^{\prime}$ with basis $\left\{x_{1}, \cdots, x_{n}\right\}$ is expressed by

$$
\left(x_{\alpha} x_{\beta}\right) x_{\gamma}=x_{\alpha}\left(x_{\beta} x_{\gamma}\right)
$$

here and throughout this section the Greek indices run from 1 to $n$. (And $\alpha$ is an index-not the multiplication of $A^{\prime}$.) Writing $x_{\alpha} x_{\beta}=$ $\sum_{\gamma} c_{\alpha \beta}^{\gamma} x_{\gamma}$, we obtain the set of structure constants $\left\{c_{\alpha \beta}^{\gamma}\right\}$, for the algebra $A^{\prime}$ with respect to the given basis $\left\{x_{1}, \cdots, x_{n}\right\}$. Associativity is expressed in terms of structure constants by

$$
\sum_{\varepsilon} c_{\alpha \beta}^{\varepsilon} c_{\varepsilon \gamma}^{\mu}=\sum_{\varepsilon} c_{\beta \gamma}^{\varepsilon} c_{\alpha \varepsilon}^{\mu}
$$

A deformation of the multiplication on $A^{\prime}$ is given by

$$
\begin{aligned}
F_{t}\left(x_{\alpha}, x_{\beta}\right) & =x_{\alpha} x_{\beta}+\sum_{i \geqq 1} f_{i}\left(x_{\alpha}, x_{\beta}\right) t^{i} \\
& =\sum_{\gamma}\left(c_{\alpha \beta}^{\gamma}+\sum_{i \geqq 1} f_{i}^{\gamma}\left(x_{\alpha}, x_{\beta}\right) t^{i}\right) x_{\gamma}
\end{aligned}
$$

Set $c_{\alpha \beta}^{\gamma}(t)=c_{\alpha \beta}^{\gamma}+\sum_{i \geqq 1} f_{i}^{\gamma}\left(x_{\alpha}, x_{\beta}\right) t^{i}$, and write $f_{0}^{\gamma}\left(x_{\alpha}, x_{\beta}\right)$ for $c_{\alpha \beta}^{\gamma}$.

The associativity condition (16) becomes in terms of the coefficients of powers of $t$, for all non-negative $p, q$, 
(18)

$$
\sum_{\varepsilon} \sum_{p+q=r} f_{p}^{\varepsilon}\left(x_{\alpha}, x_{\beta}\right) f_{q}^{\mu}\left(x_{\varepsilon}, x_{\gamma}\right)-f_{p}^{\varepsilon}\left(x_{\beta}, x_{\gamma}\right) f_{q}^{\mu}\left(x_{\alpha}, x_{\varepsilon}\right)=0
$$

Letting $a_{\alpha, \beta, p}^{\varepsilon}=f_{p}^{\varepsilon}\left(x_{\alpha}, x_{\beta}\right)$, where Greek indices run from 1 to $n$, and Latin indices from 0 to $\infty$, we rewrite (18) as

$$
\sum_{p+q=r} \sum_{\varepsilon} a_{\alpha, \beta, p}^{\varepsilon} a_{\varepsilon, \gamma, q}^{\mu}-a_{\beta, \gamma, p}^{\varepsilon} a_{\alpha, \varepsilon, q}^{\mu}=0
$$

which is a quadratic polynomial over the ground field $k$ in $n^{3}(r+1)$ variables. Denote this polynomial by $g_{\alpha, \beta, \gamma}^{\mu, r}$. Then the set $\left\{g_{\alpha, \beta, \gamma}^{\mu, r}\right\}$ indexed by $r \leqq R$ and $\alpha, \beta, \gamma, \mu$ is a finite set of polynomials, which defines an algebraic variety $G^{R}$ in $n^{3}(R+1)$-dimensional affine space. The set of varieties $\left\{G^{R}\right\}$ is ordered by inclusion, and $G^{R}$ has order of contact $R$ with the parameter space of Gerstenhaber at the point $c=\left\{c_{\alpha \beta}^{\gamma}\right\}_{\alpha, \beta, \gamma}$. In [5] Gerstenhaber takes as the parameter space for the deformation theory of $n$-dimensional associative algebras the set of structure constants for all such algebras. This corresponds to consideration of all $n$-dimensional associative algebras, each with fixed basis.

As we are interested in deformations at the point $c=\left\{c_{\alpha \beta}^{\gamma}\right\}_{\alpha, \beta, \gamma}$ of the parameter space, corresponding to $A^{\prime}$, we take $\left\{c_{\alpha \beta}^{\gamma}\right\}$ as our solution to (19) for $r=0$. Let $\left\{c_{\alpha, \beta, p}^{\gamma}\right\}_{\alpha, \beta, \gamma}^{p=0, \cdots, r-1}$ be a solution to (19) for $0 \leqq p+q=r^{\prime}<r$. Then the $r^{t h}$ obstruction to the deformation of $A^{\prime}$ is finding a solution to the system of polynomials

$$
\begin{gathered}
\sum_{\varepsilon}\left(c_{\alpha \beta}^{\varepsilon} X_{\varepsilon \gamma r}^{\mu}+X_{\alpha \beta r}^{\varepsilon} c_{\varepsilon \gamma}^{\mu}-X_{\beta \gamma r}^{\varepsilon} c_{\alpha \varepsilon}^{\mu}-c_{\beta \gamma}^{\varepsilon} X_{\alpha \varepsilon r}^{\mu}\right) \\
=\sum_{\substack{p+q=r \\
p, q>0}} \sum_{\varepsilon}\left(c_{\alpha \beta p}^{\varepsilon} c_{\varepsilon \gamma q}^{\mu}-c_{\beta \gamma p}^{\varepsilon} c_{\alpha \varepsilon q}^{\mu}\right) .
\end{gathered}
$$

This expresses the obstruction to deformation as a linear system of $n^{4}$ equations in $n^{3}$ indeterminants, and for notational simplicity, (20) may be rewritten in the form

$$
\left(\begin{array}{ccc}
a_{11} & \cdots & a_{1 M} \\
\vdots & & \vdots \\
a_{N 1} & \cdots & a_{N M}
\end{array}\right)\left(\begin{array}{c}
X_{1} \\
\vdots \\
X_{M}
\end{array}\right)=\left(\begin{array}{c}
b_{1 r} \\
\vdots \\
b_{N r}
\end{array}\right), \quad M=n^{3}, N=n^{4}
$$


where the subscript $r$ is added to the $b_{i}$ (corresponding to the quantity in parentheses on the right of (20)) to indicate their dependence upon $r$. The $a_{i j}$ are independent of $r$ (corresponding to the Hochschild coboundary operator). The condition of integrability up to order $r$, equivalently the vanishing of the $r^{\text {th }}$ obstruction, is the solvability of the system (20) for the $\left\{X_{\alpha \beta^{\gamma}}^{\mu}\right\}, r$ fixed. This condition is expressed in terms of linear algebra, by asking whether the rank of the $\left(a_{i j}\right)$ matrix is equal to the rank of $\left(a_{i j}: b_{i}\right)$-an affirmative answer being the necessary and sufficient condition for solvability.

\section{A diagram of algebras}

The previous discussion of the deformation cohomology and the Hochschild cohomology is incomplete in that no definite correlation between the two was developed. The deformation cohomology, it was pointed out, gives the automorphisms and deformations, while the Hochschild cohomology represents the "infinitesimal" automorphisms and deformations. This section and the next, in developing the final parallel to be discussed in this paper, between the analytic and algebraic theories, present an exact cohomology diagram linking the deformation and the infinitesimal cohomology theories (cf. [21, p. 68]).

Consider an associative algebra $A^{\prime}$ over a field $k$ with characteristic zero, and let $A=A^{\prime}[[t]]$. For integers $\mu \geqq-1$, we denote by $A_{\mu}$ the subset of the elements of $A$, whose first $\mu+1$ terms in their power series expansion over $A^{\prime}$ are zero. That is,

$$
A_{\mu}=\left\{a \in A: a=b_{\mu+1} t^{\mu+1}+b_{\mu+2} t^{\mu+2}+\cdots, b_{i} \in A^{\prime}\right\}
$$

The subset $A_{\mu}$ is actually an ideal in $A$, and we may consider the quotient $A^{\mu}=A / A_{\mu}$ of $A$, with respect to the obvious equivalence relation. The elements of $A^{\mu}$ will be referred to as $\mu$-jets.

One then has the exact, commutative diagram.

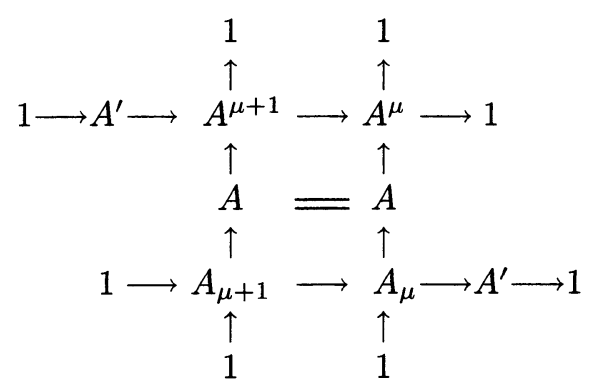

The mappings involved follow immediately from the definitions. The proofs of commutativity and exactness are straightforward. 


\section{An exact cohomology diagram}

From the above diagram (23) of algebras we obtain in a natural way the exact cohomology diagram (24). (Note that there is a considerable amount of repetition indicated in the diagram.) This section is devoted to defining the terms and the mappings in the diagram. Diagram (24) is just the expected exact cohomology diagram associated to the exact diagram (23) with the modification that the Hochschild cohomology is used where one would expect $H_{d}^{*}\left(A^{\prime}, A^{\prime}\right)$, which is not defined. Perhaps a deformation cohomology could be made meaningful for $A^{\prime}$ once a Krull topology has been introduced, enabling one to speak of $\mu$-jets, etc. We begin with a sequence of definitions, keeping in mind that the mappings are induced from (23).

$$
\begin{aligned}
& \stackrel{\rho^{1}}{\longrightarrow} H^{2}\left(A^{\prime}, A^{\prime}\right) \stackrel{\sigma^{2}}{\longrightarrow} H_{d}^{2}\left(A, A^{\mu+1}\right) \stackrel{\tau^{2}}{\longrightarrow} H_{d}^{2}\left(A, A^{\mu}\right) \stackrel{\rho^{2}}{\longrightarrow} H^{3}\left(A^{\prime}, A^{\prime}\right) \\
& \uparrow \pi_{2} \quad \uparrow \pi_{2} \\
& H_{d}^{2}(A, A)=H_{d}^{2}(A, A) \\
& \uparrow \iota_{2} \quad \uparrow \iota_{2} \\
& \stackrel{\rho_{1}}{\longrightarrow} H^{1}\left(A^{\prime}, A^{\prime}\right) \stackrel{0}{\longrightarrow} H_{d}^{2}\left(A, A_{\mu+1}\right) \stackrel{\tau_{2}}{\longrightarrow} H_{d}^{2}\left(A, A_{\mu}\right) \stackrel{\rho_{2}}{\longrightarrow} H^{2}\left(A^{\prime}, A^{\prime}\right) \\
& \left\|\quad \uparrow \lambda_{1} \quad \uparrow \lambda_{1} \quad\right\| \\
& \stackrel{0}{\longrightarrow} H^{1}\left(A^{\prime}, A^{\prime}\right) \stackrel{\sigma^{1}}{\longrightarrow} H_{d}^{1}\left(A, A^{\mu+1}\right) \stackrel{\tau^{1}}{\longrightarrow} H_{d}^{1}\left(A, A^{\mu}\right) \stackrel{\rho^{1}}{\longrightarrow} H^{2}\left(A^{\prime}, A^{\prime}\right) \stackrel{\sigma^{2}}{\longrightarrow} \\
& \uparrow \pi_{1} \quad \uparrow \pi_{1}
\end{aligned}
$$

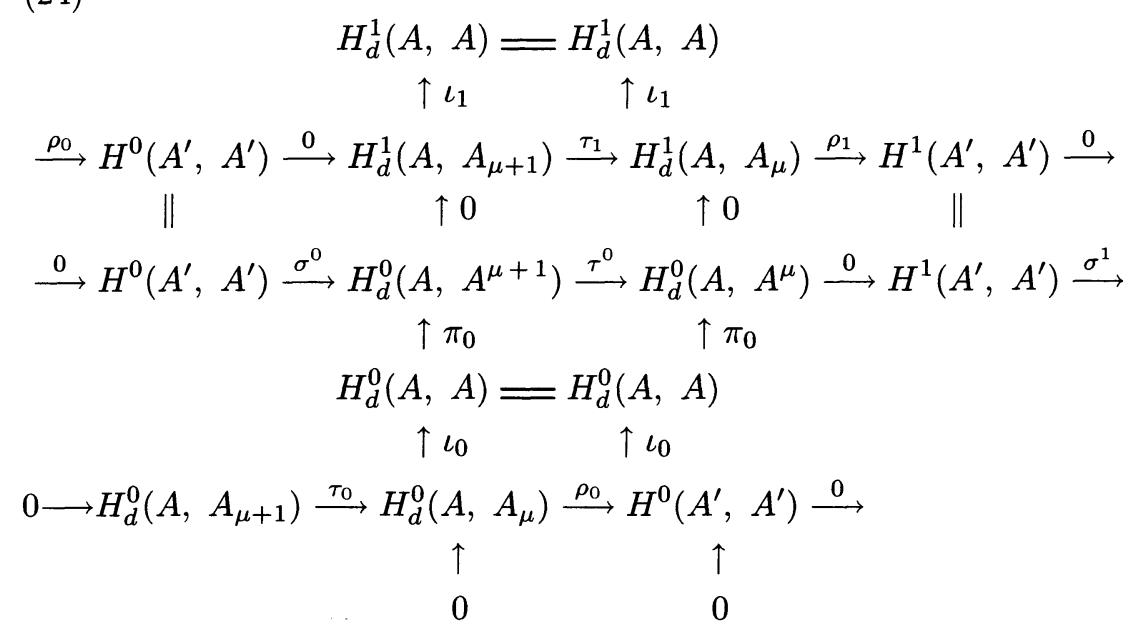

1. $C_{d}^{0}(A, A \mu)=A_{\mu}$.

2. $C_{d}^{0}(A, A \mu)=A_{\mu}$. 
And for $\mu \geqq 0$,

3. $C_{d}^{1}\left(A, A_{\mu}\right)=$ the subgroup of $C_{d}^{1}(A, A)$ of elements of the form

$$
\Phi_{\iota}=1+\sum_{i>\mu} \varphi_{i} t^{i}
$$

4. $C_{d}^{2}\left(A, A_{\mu}\right)=$ the subset of $C^{2}(A, A)$ of elements of the form

$$
F_{t}=\alpha+\sum_{i>\mu} f_{i} t^{i}
$$

5. $C_{d}^{1}\left(A, A^{\mu}\right)=$ the quotient of $C_{d}^{1}(A, A)$ by $C_{d}^{1}\left(A, A_{\mu}\right)$. Explicitly, the equivalence relation is given by

$$
\Phi_{t}=1+\sum_{i \geqq 1} \varphi_{i} t^{i} \sim \Psi_{t}=1+\sum_{i \geqq 1} \phi_{i} t^{i}
$$

if and only if $\varphi_{i}=\phi_{i}, i=1, \cdots, \mu$.

6. $C_{d}^{2}\left(A, A^{\mu}\right)=$ the quotient of $C_{d}^{2}(A, A)$ by $C_{d}^{2}\left(A, A_{\mu}\right)$, where the equivalence relation is

$$
F_{t}=\alpha+\sum_{i \geqq 1} f_{i} t^{i} \sim G_{t}=\alpha+\sum_{i \geqq 1} g_{i} t^{i}
$$

if and only if $f_{i}=g_{i}, i=1, \cdots, \mu$.

Note that $C_{d}^{1}\left(A, A^{0}\right)=\{1\}, C_{d}^{2}\left(A, A^{0}\right)=\{\alpha\}$, and $C_{d}^{q}\left(A, A_{0}\right)=$ $C_{d}^{q}(A, A), q=1,2$.

We define two differential operators as follows. On $C_{d}^{q}\left(A, A_{\mu}\right), q=$ $0,1,2, D_{\mu}$ is the restriction of the deformation coboundary operator $D$. This gives a differential complex, and, taking the cohomology complex, we obtain $H_{d}^{q}\left(A, A_{\mu}\right), q=0,1,2$. The cohomology groups may be described as of $A$.

$H_{d}^{0}\left(A, A_{\mu}\right)=A_{\mu} \cap\{$ the center of $A\}$. Recall $H_{d}^{0}(A, A)=$ the center

$H_{d}^{1}\left(A, A_{\mu}\right)=$ the cohomology classes of algebra isomorphisms of $A$ of the

$$
\text { form } \Phi_{t}=1+\sum_{i>\mu} \varphi_{i} t^{i}
$$
form

$H_{d}^{2}\left(A, A_{\mu}\right)=$ the cohomology classes of multiplications on $A$ of the

$$
F_{t}=\alpha+\sum_{i>\mu} f_{i} t^{i}
$$

The second differential operator $D^{\mu}$ is defined on representatives of $C_{d}^{q}\left(A, A^{\mu}\right), q=0,1,2$, by

i) considering $x=x_{0}+x_{1} t+\cdots+x_{u} t^{\mu}+x_{\mu+1} t^{\mu+1}+\cdots$ in $C_{\alpha}^{0}(A, A)$, let $z=x_{0}+x_{1} t+\cdots+x_{\mu} t^{\mu}$. Then $x=z+y, y \in A_{\mu}$, and define

$$
D^{\mu} x=D z
$$


ii) considering $\Phi_{t}=1+\sum_{i \geqq 1} \varphi_{i} t^{i} \in C_{d}^{1}(A, A)$, define

$$
D^{\mu} \Phi_{t}(a, b)=D \Phi_{t}(a, b)=\Phi^{-1}\left(\Phi_{a} \cdot \Phi_{b}\right) \quad \text { modulo }\left(t^{\mu+1}\right)
$$

iii) considering $F_{t}=\alpha+\sum_{i \geqq 1} f_{i} t^{i} \in C_{d}^{2}(A, A)$, define

$$
D^{\mu} F_{t}=D F_{t}=\frac{1}{2}\left[F_{t}, F_{t}\right] \quad \operatorname{modulo}\left(t^{\mu+1}\right) .
$$

Observe that

i) $D^{\mu} x=0$ if and only if $z$ lies in the center of $A$.

ii) $D^{\mu} \Phi=0$ if and only if $D^{\mu} \Phi(a, b)$ represents the trivial automorphism of $A^{\mu}$; that is, is of the form $a b+\phi_{\mu+1} t^{\mu+1}+\cdots$. This implies that

$$
\delta \varphi_{r}=-\sum_{\substack{p+q=r \\ p, q>0}} \varphi_{p} \cup \varphi_{q}, \quad r=1, \cdots, \mu .
$$

iii) $D^{\mu} F_{t}=0$ if and only if $\frac{1}{2}\left[F t, F_{t}\right]$ has the form $g_{\mu+1} t^{\mu+1}+$ $g_{\mu+2} t^{\mu+2}+\cdots$, which implies that

$$
\delta f_{r}=\sum_{\substack{p+q=r \\ p, q>0}} f_{p} * f_{q}, \quad r=1, \cdots, \mu .
$$

We shall refer to $F_{t}$ as a multiplication modulo $t^{\mu+1}$.

It follows immediately from the definitions that $D^{\mu} \cdot D^{\mu}=0$, and that $D^{\mu}$ is a well-defined differential operator on the cochains $C_{d}^{q}\left(A, A^{\mu}\right), q=$ $0,1,2$, as the definitions are independent of the choice of representative elements of the equivalence classes. Cocycles in $Z_{d}^{1}\left(A, A^{\mu}\right)$ and $Z_{d}^{2}\left(A, A^{\mu}\right)$ will be referred to as $\mu$-jets of automorphism and of deformation, respectively.

As the proof of the exactness and commutativity of diagram (24) follows immediately from the mappings involved, it suffices to give explicit description of these mappings. The mappings are described most easily by representatives and/or on cocycles. It is a simple exercise to see that they are well-defined on the appropriate cohomology classes.

The mapping $\rho^{2}$ is the $(\mu+1)^{t h}$ obstruction map defined on a representative by

$$
\rho^{2}\left(F_{t}=\alpha+\sum_{i>1} f_{i} t^{i}\right) \text { is the class of } \sum_{\substack{p+q=\mu+1 \\ p, q>0}} f_{p} * f_{q}
$$

Similarly, $\rho^{1}$ is the $(\mu+1)^{t h}$ obstruction to the integration of an infinitesimal automorphism.

Defined on cocycles, the maps, $\tau_{0}, \tau_{1}, \tau_{2}, \iota_{0}, \iota_{1}, \iota_{2}$ are inclusions.

The mapping $\sigma^{2}$ is induced from the map of cocycles given by

$$
f \in Z^{2}\left(A^{\prime}, A^{\prime}\right) \rightarrow F_{t}=\alpha+f t^{\mu+1}
$$


Similarly, $\sigma^{1}$ and $\sigma^{0}$ are induced from the maps of cocycles given respectively by

$$
\begin{aligned}
& \varphi \in Z^{1}\left(A^{\prime}, A^{\prime}\right) \rightarrow \Phi_{t}=1+\varphi t^{\mu+1} \\
& x \in Z^{0}\left(A^{\prime}, A^{\prime}\right) \rightarrow x t^{\mu+1}
\end{aligned}
$$

An element of $H_{d}^{2}\left(A, A_{\mu}\right)$ may be represented by

$$
F_{t}=\alpha+f_{\mu+1} t^{\mu+1}+\cdots, \text { where } \frac{1}{2}\left[F_{t}, F_{t}\right]=0 .
$$

Define $\rho_{2}\left(F_{t}\right)$ to be the class of $f_{\mu+1}$, which by Proposition 8 is a cocycle. If $f_{\mu+1} \in B^{2}\left(A^{\prime}, A^{\prime}\right)$, then $\rho_{2}\left(F_{t}\right)=0$, and $F_{t}$ represents an element of $H_{d}^{2}\left(A, A_{\mu+1}\right)$. Thus $\rho_{2}$ is well-defined on cohomology classes. Analogously, $\rho_{1}$ and $\rho_{0}$ are defined respectively from the maps

$$
\begin{aligned}
& \Phi_{t}=1+\varphi_{\mu+1} t^{\mu+1}+\cdots \rightarrow \varphi_{\mu+1} \\
& a=\sum_{i>\mu} a_{i} t^{i} \rightarrow a_{\mu+1} .
\end{aligned}
$$

The connecting homomorphism $\lambda_{1}$ is defined on representatives of the $\mu$-jets of automorphism by

$$
\lambda_{1}\left(\Phi_{t}\right)(a, b)=\Phi^{-1}\left(\Phi_{a} \cdot \Phi_{b}\right)
$$

where $\Phi_{t}=1+\sum_{i \geqq 1} \varphi_{i} t^{i}$ and $\left\{\Phi_{t}\right\} \in Z_{d}^{1}\left(A, A^{\mu}\right)$. To prove that $\lambda_{1}$ is well defined on $Z_{d}^{1}\left(A, A^{\mu}\right)$, we must show that $\Phi^{-1}\left(\Phi_{a} \cdot \Phi_{b}\right)$ and $\Psi^{-1}\left(\Psi_{a} \cdot \Psi_{b}\right)$ are cohomologous in $Z_{d}^{2}\left(A, A_{\mu}\right)$, when $\Phi-\Psi=0$ modulo $t^{\mu+1}$. Letting $\Psi_{t}=1+\sum_{i \geqq 1} \varphi_{i} t^{i}$, set $F_{t}(a, b)=\Phi^{-1}\left(\Phi_{a} \cdot \Phi_{b}\right), G_{t}(a, b)=\Psi^{-1}\left(\Psi_{a} \cdot \Psi_{b}\right)$. We have then to prove the existence of a $\Delta=1+\sum_{i>\mu} \sigma_{i} t^{i}$ such that

$$
\Delta_{t}\left(F_{t}(a, b)\right)=G_{t}\left(\Delta_{t}(a), \Delta_{t}(b)\right)
$$

The reader may verify that $\Delta_{t}=\Psi^{-1}\left(\Phi_{t}\right)$ satisfies (31).

\section{Consequences of the diagram}

Let $H^{3}\left(A^{\prime}, A^{\prime}\right)$ be the zero group. Then for all $\mu, H_{d}^{2}\left(A, A^{\mu+1}\right) \stackrel{\tau^{2}}{\longrightarrow}$ $H_{d}^{2}\left(A, A^{\mu}\right)$ is onto, stating that every class of deformation cochains satisfying the associativity condition up to order $\mu$ also satisfies it up to order $\mu+1$. As $\mu$ runs from 1 to $\infty$, this says that all obstructions vanish.

Suppose $H^{2}\left(A^{\prime}, A^{\prime}\right)=0$. It was observed as a corollary to Proposition 8 that under this assumption $A^{\prime}$ is rigid, i.e., $H_{d}^{2}(A, A)=0$. This result also follows immediately from (24). From the third row we have 


$$
0 \longrightarrow H_{d}^{2}\left(A, A_{\mu+1}\right) \stackrel{\tau_{2}}{\longrightarrow} H_{d}^{2}\left(A, A_{\mu}\right) \longrightarrow 0
$$

and by recursion

$$
H_{d}^{2}\left(A, A_{\mu+\eta}\right) \simeq H_{d}^{2}\left(A, A_{\mu}\right)
$$

for nonnegative $\mu$ and $\eta$. Setting $\mu=0$,

$$
H_{d}^{2}\left(A, A_{\eta}\right) \simeq H_{d}^{2}(A, A)
$$

which implies $H_{d}^{2}(A, A)=0$.

We further observe that

$$
0 \longrightarrow H_{d}^{2}\left(A, A^{\mu+1}\right) \longrightarrow H_{d}^{2}\left(A, A^{\mu}\right)
$$

is exact for all $\mu \geqq 0$, and composing injections,

$$
0 \longrightarrow H_{d}^{2}\left(A, A^{\mu+\eta}\right) \longrightarrow H_{d}^{2}\left(A, A^{\mu}\right)
$$

is exact for all nonnegative $\mu$ and $\eta$. In particular, as $C_{d}^{2}\left(A, A_{0}\right)=$ $C_{d}^{2}(A, A), H_{d}^{2}\left(A, A^{0}\right)=0$, and therefore $H_{d}^{2}\left(A, A^{\eta}\right)=0$ for all $\eta \geqq 0$. This also implies the rigidity.

Similar observations can be made about the vanishing of $H^{1}\left(A^{\prime}, A^{\prime}\right)$ and $H^{2}\left(A^{\prime}, A^{\prime}\right)$ with respect to automorphisms.

The diagram (24) was expressed for arbitrary nonnegative $\mu$. Specializing to $\mu=0$, we obtain the isomorphisms

$$
\begin{aligned}
& H_{d}^{2}\left(A, A^{1}\right) \simeq H^{2}\left(A^{\prime}, A^{\prime}\right) \\
& H_{d}^{1}\left(A, A^{1}\right) \simeq H^{1}\left(A^{\prime}, A^{\prime}\right)
\end{aligned}
$$

PART II

FINITELY MANY OBSTRUCTIONS AND THE DEFORMATION OF MODULES

The twofold object of Part II is i) to show that there are only finitely many obstructions to the extension of a Hochschild second cohomology class in $H^{2}(A, M)$, where $M$ is a two-sided module over an associative algebra $A$. The proof will be seen to rest on a certain linearity condition-a condition which fails when discussing deformations of $A$. And ii) to develop the theory of deformations of two-sided modules over associative algebras. The latter parallels the deformation of complex analytic fiber bundles.

Consider $M$, a two-sided $A$-module, $A$ being an associative algebra, and let 
$C_{A}=C(A, A)$, the Hochschild cochains of $A$ with coefficients in $A$, with Hochschild coboundary $\delta_{A}$. After a change from the usual grading, we shall denote this complex by $A^{*}$.

$C_{M}=C(A, M)$, the Hochschild cochains of $A$ with coefficients in $M$, with Hochschild coboundary $\delta_{M}$. Later this complex is denoted by $M^{*}$.

\section{2. $M^{*}$ is a complex over the Lie algebra complex $A^{*}$}

Clearly, $\left(C_{A}, \delta_{A}\right)$ and $\left(C_{M}, \delta_{M}\right)$ are graded differential complexes. We first recall the graded Lie algebra structure of $\left(C_{A}, \delta_{A}\right)$ and then show that $\left(C_{M}, \delta_{M}\right)$ may be considered as a graded module over the graded Lie algebra $\left(C_{A}, \delta_{A}\right)$. It is customary to write $C^{p}(A, A)=\operatorname{Hom}\left(T_{p}(A), A\right)$. However, in discussing the graded structure it is necessary to have $\operatorname{deg} f=p-1$, for $f \in C^{p}(A, A)$, and similarly for the cochains $C(A, M)$. To simplify the notation, especially with respect to this concept of degree, we write $A^{p}$ for $C_{A}^{p+1}$ and $M^{p}$ for $C_{M}^{p+1}$. Thus we consider the complexes $\left(A^{*}, \delta_{A}\right)$ and $\left(M^{*}, \delta_{M}\right)$. The arguments of $\S 13$ are made easier if we know that $M^{*}$ is a complex over $A^{*}$. This will be demonstrated now.

For $f \in A^{p}, g \in A^{q},[f, g] \in A^{p+q}$ is given by

$$
[f, g]=f * g-(-1)^{p q} g * f \text {, }
$$

where

$$
\begin{gathered}
f * g\left(a_{0}, \cdots, a_{p+q}\right) \\
=\sum_{i \geq 0}(-1)^{i q} f\left(a_{0}, \cdots, a_{i-1}, g\left(a_{i}, \cdots, a_{i+q}\right), a_{i+q+1}, \cdots, a_{p+q}\right) .
\end{gathered}
$$

The reader may prove that the bracket so defined satisfies the Jacobi identity and is compatible with the Hochschild coboundary operator. Hence, $\left(A^{*}, \delta_{A}\right)$ is a graded Lie algebra complex, with $\delta_{A}$ a right inner derivation of of degree +1 .

To give the two-sided module structure of $\left(M^{*}, \delta_{M}\right)$ over $\left(A^{*}, \delta_{A}\right)$ define $A^{p} \otimes M^{q} \rightarrow M^{p+q}$ by

$$
[f, g]=-(-1)^{p q} g * f, \text { for } f \in A^{p}, g \in M^{q},
$$

and $M^{q} \otimes A^{p} \rightarrow M^{p+q}$ by

$$
[g, f]=g * f, \text { for } f \in A^{p}, g \in M^{q} .
$$

In order that this structure actually be that of a two-sided module, it is necessary that

$$
\begin{gathered}
{[[h, f], g]+(-1)^{p q+1}[[h, g], f]=[h,[f, g]]} \\
{[f,[g, h]]+(-1)^{p q+1}[g,[f, h]]=[[f, g], h]}
\end{gathered}
$$


hold for $f \in A^{p}, g \in A^{q}, h \in M^{*}$. Note carefully that there are two different brackets appearing in equations (35) and (36). This should cause no difficulty as $f * h$ is not defined in general for $f \in A^{*}, h \in M^{*}$. The following relation (37) is used in verifying (35) and (36). The proofs are left to the reader.

$$
(h * g) * f+(-1)^{p q+1}(h * f) * g=h *(g * f)+(-1)^{p q+1} h *(f * g)
$$

for $f \in A^{p}, g \in A^{q}, h \in M^{*}$.

Finally, to confirm that $\left(M^{*}, \delta_{M}\right)$ as a graded complex lies over the graded complex $\left(A^{*}, \delta_{A}\right)$, it is necessary to verify, for $f \in A^{p}, g \in M^{q}$, that

$$
\begin{gathered}
\delta_{M}[f, g]=(-1)^{q}\left[\delta_{A} f, g\right]+\left[f, \delta_{M} g\right] \\
\delta_{M}[g, f]=(-1)^{p}\left(\delta_{M} g, f\right]+\left[g, \delta_{A} f\right]
\end{gathered}
$$

One proves

$$
\begin{gathered}
\delta_{A} f=-[f, \alpha] \\
\delta_{M} g=-g * \alpha+(-1)^{q} \pi * g
\end{gathered}
$$

where $\alpha$ is the multiplication of $A$, and $\pi$ reflects the given $A$-module structure of $M$. That is, $\pi: A \otimes M \rightarrow M, \pi: M \otimes A \rightarrow M$. Equations (38) and (39) follow from the fact that the Hochschild coboundary operator on the cochains of an algebra with values in itself is a right derivation on the graded Lie algebra of cochains. That is,

$$
\delta[f, g]=(-1)^{q}[\delta f, g]+[f, \delta g], \text { where } f \in Q^{p}, g \in Q^{q},
$$

for an arbitrary cochain complex $Q$ of an algebra with values in itself. The remainder of the proof of (38) and (39) may be found as a part of Theorem 4 in $[3$, p. 282].

\section{Finitely many obstructions}

One of the outstanding problems of deformation theory is that of finitely many obstructions. One conjectures an affirmative answer in the algebraic case. Similarly, Grauert has conjectured such an answer for the deformation of complex analytic structures. This section deals with an integration or extension problem in which the obstructions are not quadratic, as in the above conjectures, but instead simply linear. Namely, there are only finitely many obstructions to extending a class in $H^{r+1}(A, M)$. The proof suggested by Griffiths [8] relies on earlier work by Grauert, Kodaira, and Spencer. The following discussion presented in a series of lemmas is valid for any graded differential complex over a graded Lie algebra, although our interest is in cochain complexes of algebras and modules. We shall have occasion to refer to rings of formal power series $M^{*}[[t]]$ and $A^{*}[[t]]$, the quotient field $M^{*}((t))$, and $A^{*}\{t\}$, the ideal in $A^{*}[[t]]$ of formal power series with zero constant term. 
Consider $\Phi_{t} \in A^{1}\{t\}$, such that

$$
\delta_{A} \Phi_{t}=\frac{1}{2}\left[\Phi_{t}, \Phi_{t}\right]
$$

and define $\delta_{\Phi}: M^{q}[[t]] \rightarrow M^{q+1}[[t]]$ by

$$
\delta_{\Phi} F_{t}=\delta_{M} F_{t}-\left[\Phi_{t}, F_{t}\right]
$$

One checks that $\delta_{\Phi} \cdot \delta_{\Phi}=0$, and hence $\left(M^{*}[[t]], \delta_{\Phi}\right)$ is again a differential complex.

An extension of a Hochschild $(r+1)$-cocycle $f \in M^{r}$, with respect to $\Phi_{t} \in A^{1}\{t\}$, is an element $F_{t}$ in $M^{r}[[t]]$ such that i) $F_{0}=f$, ii) $\delta_{\Phi} F_{t}=0$. The cocycle $f$ is said to be extendible if such an extension $F_{t}$ exists. All $(r+1)$-coboundaries $g=\delta h$ are extendible. $\left(G_{t}=\delta_{\Phi} h\right.$ is an extension of $g$ ). Thus, the question of extendibility depends upon the cohomology class of $f$ in $H^{r+1}(A, M)$. A cohomology class is said to be extendible if any (and therefore all) representing cocycle is extendible.

Given an extension $F_{t}=\sum_{i \geqq 0} f_{i} t^{i}$, of $f=f_{0}$, one has from (33) the following set of relations :

$$
\begin{aligned}
\delta_{M} f_{i} & =\sum_{\substack{p+q=i \\
p>0}}\left[\varphi_{p}, f_{q}\right], \text { where we have written } \Phi_{t}=\sum_{i \geqq 1} \varphi_{i} t^{i} \\
& =(-1)^{r} \sum_{\substack{p+q=i \\
p>0}} f_{q} * \varphi_{p} \quad \text { for all } i \geqq 1
\end{aligned}
$$

or using (41),

$$
(-1)^{r} \pi * f_{i}-f_{i}-f_{i} * \alpha=(-1)^{r} \sum_{\substack{p+q=i \\ p>0}} f_{q} * \varphi_{p}
$$

Suppose one is able to find $f_{0}, \cdots, f_{N-1}$ such that $(44)_{i}$ is satisfied for $i=1, \cdots, N-1$; that is, $f_{0}$ can be extended up to order $N-1$, then

$$
\omega_{N}=\sum_{\substack{p+q=N \\ p>0}}\left[\varphi_{p}, f_{q}\right]
$$

is said to be the $N^{t h}$ obstruction to the extension of $f=f_{0}$. The result is that there are only finitely many obstructions in the sense that there exists an $N$ such that if $f$ is extendible to order $N$ (i.e., $\omega_{N}$ is cohomologous to zero), then $f$ is extendible. This conclusion is based on a series of four lemmas whose proofs may be found in [8]. 
Denote the $r$-cocycles of $\left(M^{*}[[t]], \delta_{\Phi}\right)$ by $Z_{\Phi}^{r}(M)$. If $F_{t} \in Z_{\Phi}^{t}(M)$ extends $f \in Z^{r+1}(A, M)$ (note the change in grading), then $F_{t}$ is called a jump extension if there is a $G_{t}$ in $M^{r-1}((t))$ with $\delta_{\Phi} G_{t}=F_{t}$ (i.e., $G_{t}$ has the form $\sum_{i=-N}^{\infty} g_{i} t^{i}, g_{i} \in C^{r}(A, M)$, for some nonnegative $\left.N\right)$. And $f \in Z^{r+1}(A, M)$ is called a jump cocycle if there is a jump extension $F_{t}$ of $f$. A class in $H^{r+1}(A, M)$ is called a jump class if any representative is a jump cocycle. Since $\delta_{\Phi} g$ is a jump extension of $\delta_{M} g$, the notion of jump class is well defined.

Lemma 3. The $N^{\text {th }}$ obstruction $\omega_{N}$ to extension is in $Z^{r+2}(A, M)$.

Lemma 4. $\omega \in Z^{r+2}(A, M)$ is a jump cocycle if and only if $\omega$ is an obstruction to extending some $f$ in $Z^{r+1}(A, M)$.

Define $N(\omega)$ to be the least integer $n$, such that $\omega$ is an $n^{\text {th }}$ obstruction to the extension of some $f$ in $Z^{r+1}(A, M)$. Given $f$ in $Z^{r+1}(A, M)$, let $N(f)$ be the greatest integer $n$ such that $f$ can be extended up to order $n-1$. In other words, we can find $f_{0}=f, f_{1}, \cdots f_{\mu}$, satisfying $(44)_{i}$ for $i=0, \cdots, \mu$ when $\mu=n-1$, but not when $\mu=n$.

Comparing the obstructions to the integration of Hochschild cocycles in $Z^{2}(A, A)(8)$ and of 1-cocycles in $Z^{1}(A, A)(11)$, with the obstruction (45) to the extension of a cocycle in $Z^{r+1}(A, M)$ with respect to $\Phi_{t} \in A^{1}\{t\}$, we see that the obstruction is quadratic in the $\left\{f_{i}\right\}$ in the previous cases, whereas it is linear in the $\left\{f_{i}\right\}$ in the present context. The distinction between the situations with linear and quadratic obstructions is made clear in the following lemma. Because the proof does not hold for quadratic obstructions, this lemma is not proven for the case of algebra deformations, and we are presently unable to obtain the statement of finitely many obstructions for algebra deformations or automorphisms. Finally, we remark that the other lemmas of this section do hold for deformations.

Lemma 5. i) If $\omega$ is an obstruction of order $N(\omega)$ to the extension of $f \in Z^{r+1}(A . M), N(\omega)=N(f)$.

ii) If $\omega$ is an obstruction of order $N(f)$ to the extension of $f \in$ $Z^{r+1}(A, M), N(\omega)=N(f)$.

The first statement says that if $\omega$ is an $n^{\text {th }}$ obstruction to the extension of some $f \in Z^{r+1}(A, M)$, and if $f$ is extendible to order $n+1$, then $\omega$ is an $(n-1)^{t h}$ obstruction for some cocycle in $Z^{r+1}(A, M)$.

Part ii) says that if $f$ is extendible only to order $n$, and if $\omega$ is an $n^{\text {th }}$ obstruction to the extension of $f$, then $\omega$ is not an $m^{\text {th }}$ obstruction to the extension of any $g \in Z^{r+1}(A, M)$ for $m<n$.

Under the assumption that $H^{r+2}(A, M)$ is finite dimensional, the subset of jump classes (equal the subset of obstructions) is finite dimensional over $k$. Let $\omega_{1}, \cdots, \omega_{s}$ be a basis for a subset of obstructions. To each $\omega_{i}$ associate $\Psi_{t}^{i} \in M^{r+1}[[t]]$, and $G_{t}^{i} \in M^{r}((t))$ such that
i) $\Psi_{0}^{i}=\omega_{i}$,
ii) $\delta_{\Phi} G_{t}^{i}=\Psi_{t}^{i}$,
iii) $G_{t}^{i}$ has a pole of order $N\left(\omega_{i}\right)$ at $t=0$. 
Let $N_{r+1}=\max \left\{N\left(\omega_{i}\right): 1 \leqq i \leqq s\right\}$. Corresponding to a linear (over $k$ ) combination $\omega$ of obstructions $\left\{\omega_{1}, \cdots, \omega_{s}\right\}$ is a linear (over $k$ ) combination $G_{t}$ of formal power series $\left\{G_{t}^{1}, \cdots, G_{t}^{s}\right\}$. Since the order of the pole of $G_{t}$ at $t=0$ is not greater than the maximum of the orders of the poles $G_{t}^{i}, 1=1, \cdots, s$ at $t=0, N(\omega) \leqq N_{r+1}$.

Lemma 6. Suppose $f \in Z^{r+1}(A, M)$ has for some $n \geqq N_{r+1}$ an extension of order $n$. Then $f$ is extendible.

When $A$ is finite dimensional over $k$ and $M$ is finite dimensional over $A, H^{r+2}(A, M)$ is finite dimensional, and we conclude from the preceding four lemmas:

Theorem 3. There are only finitely many obstructions to extending a class in $H^{r+1}(A, M)$.

Let $\Phi_{t}=\alpha t$. Such a $\Phi_{t}$ satisfies (43), as $\alpha$ is the coboundary of the identity automorphism of $A$, and therefore $\delta_{A} \alpha=0$. Applying the preceding results, we have that given $f \in Z^{r+1}(A, M)$, there exist only finitely many obstructions to obtaining $F_{t}=\sum_{i \geqq 0} f_{i} t^{i}, f_{0}=f$, such that $\delta f_{i}=(-1)^{r} f_{i-1} * \alpha$. For $r=1$, this becomes

$$
\delta f_{i}=f_{i-1}(a, b c)-f_{i-1}(a b, c) .
$$

A more substantial result is obtained by taking $\Phi_{t}=\sum_{i \geqq 1} \varphi_{i} t^{i}$, where $\alpha+\sum_{i \geqq 1} \varphi_{i} t^{i} \in Z_{d}^{2}(A, A)$. In the case, (43) is just the wellknown deformation condition (6) and is satisfied. Consequently, given $f \in Z^{2}(A, M)$, there are at most finitely many obstructions to the extension of $f$ to $F_{t}=\sum_{i \geqq 0} f_{i} t^{i}$ such that

$$
\delta f_{i}=-\sum_{\substack{p+q=i \\ p>0}} f_{q} * \varphi_{p}
$$

or more generally, given $f \in Z^{r+1}(A, M)$, there are finitely many obstructions to obtaining $F_{t}=\sum_{i \geqq 0} f_{i} t^{i}$, where $f_{0}=f$, and

$$
\delta f_{i}=(-1)^{r} \sum_{\substack{p+q=i \\ p>0}} f_{q} * \varphi_{p}
$$

\section{Module cohomology}

Consideration of the deformation theory for associative algebras and Hochschild cohomology and for Lie algebras and Chevalley-Eilenberg cohomology suggests that a well-defined deformation determines a cohomology theory when one requires the infinitesimal deformations to be classes. The situation for analytic structures and commutative algebras supports this view. When certain homological hypotheses are satisfied, 
such a cohomology theory is unique. That is, knowledge of the 0 - and 1cohomology uniquely determines the $q$-cohomology. We now apply this philosophy to the case of modules. By first describing the deformation of a two-sided module $M$ over an algebra $A$, we are able to define a cohomology, whose cochains are maps of $T(A) \otimes M \otimes A$ into $M$, which might be called the cohomology of $M$ over $A$ with values $M$.

Let $M$ be a two-sided $A$-module, with external operation $\pi$, and let $\Phi_{t}=\sum_{i \geqq 0} \varphi_{i} t^{i}$ be a deformation of $A$. Then $F_{t}=\sum_{i \geqq 0} f_{i} t^{i}$, where $f_{0}=\pi$ is called a deformation of the two-sided module structure with respect to $\Phi_{t}$ if, for $a, b \in A, m \in M$,

$$
\begin{aligned}
& F_{t}\left(\Phi_{t}(a, b), m\right)=F_{t}\left(a, F_{t}(b, m)\right), \\
& F_{t}\left(F_{t}(m, a), b\right)=F_{t}\left(m, \Phi_{t}(a, b)\right), \\
& F_{t}\left(F_{t}(a, m), b\right)=F_{t}\left(a, F_{t}(m, b)\right),
\end{aligned}
$$

where (47), (48), and (49) generalize $(a b) m=a(b m),(m a) b=m(a b)$, and $(a m) b=a(m b)$, respectively. We use $F_{t}$ to denote the change in both the left and the right module structure. Which multiplication is meant at a given time will be clear from the order of the arguments.

Expanding (47)-(49) in powers of $t$, one obtains for all $r$ and for $p$ and $q \geqq 0$ :

$$
\begin{gathered}
\sum_{p+q=r} f_{p}\left(\varphi_{q}(a, b), m\right)-f_{p}\left(a, f_{q}(b, m)\right)=0 \\
\sum_{p+q=r} f_{p}\left(f_{q}(m, a), b\right)-f_{p}\left(m, \varphi_{q}(a, b)\right)=0 \\
\sum_{p+q=r} f_{p}\left(f_{q}(a, m), b\right)-f_{p}\left(a, f_{q}(m, b)\right)=0 .
\end{gathered}
$$

We now wish to define the coboundary $d f$ of a homomorphism $f$ : $A \otimes M \rightarrow M$ as a step towards introducing the cohomology of $M$ over $A$ with coefficients in $A$. For any homomorphism $f: A \otimes M \rightarrow M$ or $f: M \otimes A \rightarrow M$, define, for $a, b \in A, m \in M$,

$$
d f(a, m, b)=a f(m, b)-f(a m, b)+f(a, m b)-f(a, m) b .
$$

With this definition for $d,(52)$ can be rewritten for nonnegative $r$ as

$$
d f_{r}(a, m, b)=\sum_{\substack{p+q=r \\ p, q>0}} f_{p}\left(f_{q}(a, m), b\right)-f_{p}\left(a, f_{q}(m, b)\right)
$$

and as a consequence, $d f_{1}(a, m, b)=0$. 
Consider $K^{r}=\operatorname{Hom}\left(T_{r}(A) \otimes M, M\right) \oplus \operatorname{Hom}\left(T_{r-1}(A) \otimes M \otimes A, M\right)$, and define $d: K^{r} \rightarrow K^{r+1}$ by, for $r=0$

$$
d f(a, m)=a f(m)-f(a m), \quad d f(m, b)=-f(m b)+f(m) b
$$

for $r=1$ and $f: A \otimes M \rightarrow M$ or $f: M \otimes A \rightarrow M$

$$
d f(a, m, b)=a f(m, b)-f(a m, b)+f(a, m b)-f(a, m) b
$$

for $r \geq 2$ define

$$
\begin{aligned}
& d f\left(a_{0}, \cdots, a_{r}, m\right)=a_{0} f\left(a_{1}, \cdots, a_{r}, m\right) \\
& \quad+\sum_{i=1}^{r}(-1)^{i} f\left(a_{0}, \cdots, a_{i-2}, a_{i-1} a_{i}, a_{i+1}, \cdots, a_{r}, m\right) \\
& \quad+(-1)^{r+1} f\left(a_{0}, \cdots, a_{r-1}, a_{r} m\right)
\end{aligned}
$$

for $f: T_{r}(A) \otimes M \rightarrow M$, and

$$
\begin{aligned}
d f\left(a_{0}, \cdots,\right. & \left.a_{r-1}, m, a_{r}\right)=a_{0} f\left(a_{1}, \cdots, a_{r-1}, m\right) \\
& +\sum_{i=1}^{r-1}(-1)^{i} f\left(a_{0}, \cdots, a_{i-2}, a_{i-1} a_{i}, a_{i+2}, \cdots, a_{r-1}, m, a_{r}\right) \\
& +(-1)^{r} f\left(a_{0}, \cdots, a_{r-2}, a_{r-1} m, a_{r}\right) \\
& +(-1)^{r+1} f\left(a_{0}, \cdots, a_{r-1}, m a_{r}\right)+(-1)^{r} f\left(a_{0}, \cdots, a_{r-1}, m\right) a_{r}
\end{aligned}
$$

for $f: T_{r-1}(A) \otimes M \otimes A \rightarrow M$. The differential operator $d$ so defined satisfies (53) when $f$ is defined on either $M \otimes A$ or $A \otimes M$. The involved computations to prove $d^{2}=0$ are omitted. Considering the cohomology of $\left(K^{*}, d\right)$, we have a cohomology theory in which the 1-cocycles are infinitesimal deformations of the two-sided module structure of $M$ over A.

As the $r^{\text {th }}$ obstruction, which is the module cohomology class of the righthand side of (54), to the integration of $f_{1}$ is quadratic in the $f_{i}$, the proof of finitely many obstruction in $\S 13$ fails for module deformations, just as it failed for algebra deformations. It will be seen in $\S \S 15$ and 16 that these two questions are not independent.

\section{Compatibility of deformations}

This section considers simultaneously an associative algebra $A$; a twosided $A$-module $M$; a deformation $\Phi_{t}=\sum_{i \geqq, 0} \varphi_{i} t^{i}$ of $A$; a deformation $F_{t}=\sum_{i \geqq 0} f_{i} t^{i}$ of the two-sided $A$-module structure of $M$ with respect to $\Phi_{t} ;$ and an extension $G_{t}=\sum_{i \geqq 0} g_{i} t^{i}$ of a 2-cocycle $g$ in $Z^{2}(A, M)$ with respect to $\Phi_{t}$, as in $\S 13$. The result of this section is that we obtain in a natural way an algebra and a deformation of this algebra when $F_{t}$ and $G_{t}$ satisfy a relation expressing compatibility. 
First, recall the deformation conditions, for all $r \geqq 0$, where $p, q \geqq 0$,

$$
\begin{array}{r}
\sum_{p+q=r} \varphi_{p}\left(\varphi_{q}(a, b), c-\varphi_{p}\left(a, \varphi_{q}(b, c)\right)=0,\right. \\
\sum_{p+q=r} f_{p}\left(\varphi_{q}(a, b), m\right)-f_{p}\left(a, f_{q}(b, m)\right)=0, \\
\sum_{p+q=r} f_{p}\left(m, \varphi_{q}(a, b)\right)-f_{p}\left(f_{q}(m, a), b\right)=0, \\
\sum_{p+q=r} f_{p}\left(a, f_{q}(m, b)\right)-f_{p}\left(f_{q}(a, m), b\right)=0, \\
\pi * g_{r}(a, b, c)+\sum_{p+q=r} g_{p}\left(a, \varphi_{q}(b, c)\right)-g_{p}\left(\varphi_{q}(a, b), c\right)=0 .
\end{array}
$$

Equation (55) is the deformation condition for the algebra $A$. Equations (56) and (57) give the conditions for the deformation of the left and right module structure of $M$ over $A$ with respect to the algebra deformation $\Phi_{t}$, while (58) is the two-sided module deformation relation. Equation (59) is the same as (46) for $r=1$.

Consider the vector space direct sum of $A$ and $M$, denoted by $A+M$, where a multiplication on $A+M$ is given by

$$
\Psi_{t}\left(x_{1}, x_{2}\right)=\left(\Phi_{t}\left(a_{1}, a_{2}\right), F_{t}\left(a_{1}, m_{2}\right)+F_{t}\left(m_{1}, a_{2}\right)+G_{t}\left(a_{1}, a_{2}\right)\right)
$$

and $x_{i}=a_{i}+m_{i}, a_{i} \in A, m_{i} \in M$ for $i=1,2$. In order that $\Psi_{t}\left(x_{1}, x_{2}\right)=\sum_{i \geqq 0} \varphi_{i}\left(x_{1}, x_{2}\right) t^{i}$ be an associative multiplication, it is necessary and sufficient that

$$
\phi_{i}\left(x_{1}, x_{2}\right)=\sum_{\substack{p+q=i \\ p, q \geqq 0}}\left(\varphi_{p}\left(a_{1}, a_{2}\right), f_{q}\left(a_{1}, m_{2}\right)+f_{q}\left(m_{1}, a_{2}\right)+g_{q}\left(a_{1}, a_{2}\right)\right)
$$

satisfy the associative deformation condition (55).

Theorem 4. $\Psi_{t}$ defined by (60) gives a deformation of the algebra $(A+M)_{g}$, which has multiplication given by

$$
(a, m)\left(a^{\prime}, m^{\prime}\right)=\left(a a^{\prime}, a m^{\prime}+m a^{\prime}+g\left(a, a^{\prime}\right)\right)
$$

if and only if the extension of $g \in Z^{2}(A, M)$ is compatible, in the sense of $(66)$, with the deformation of the module structure.

The significance of the compatibility requirement will be made clear in the course of the proof, which is a straightforward verification of (55), using (61). One has 


$$
\begin{aligned}
& \sum_{\substack{i+j=\mu \\
i, j \geqq 0}} \phi_{i}\left(\phi_{j}\left(x_{1}, x_{2}\right), x_{3}\right)-\phi_{i}\left(x_{1}, \phi_{j}\left(x_{2}, x_{3}\right)\right) \\
& =\left(\sum _ { \substack { p + q + r + s = \mu \\
p , q , r , s \geqq 0 } } \left[\varphi_{r}\left(\varphi_{p}\left(a_{1}, a_{2}\right), a_{3}\right)-\varphi_{r}\left(a_{1}, \phi_{q}\left(a_{2}, a_{3}\right)\right]\right.\right. \\
& \sum_{\substack{p+q+r+s=\mu \\
p, q, r, s \geqq 0}}\left[f_{s}\left(f_{q}\left(a_{1}, m_{2}\right), a_{3}\right)-f_{s}\left(a_{1}, f_{q}\left(m_{2}, a_{3}\right)\right)\right. \\
& \quad+f_{s}\left(\varphi_{p}\left(a_{1}, a_{2}\right), m_{3}\right)-f_{s}\left(a_{1}, f_{q}\left(a_{2}, m_{3}\right)\right) \\
& \quad+f_{s}\left(f_{q}\left(m_{1}, a_{2}\right), a_{3}\right)-f_{s}\left(m_{1}, \varphi_{p}\left(a_{2}, a_{3}\right)\right) \\
& \quad+g_{s}\left(\varphi_{p}\left(a_{1}, a_{2}\right), a_{3}\right)-g_{s}\left(a_{1}, \varphi_{p}\left(a_{2}, a_{3}\right)\right) \\
& \left.\left.\quad+f_{s}\left(g_{q}\left(a_{1}, a_{2}\right), a_{3}\right)-f_{s}\left(a_{1}, g_{q}\left(a_{2}, a_{3}\right)\right)\right]\right) .
\end{aligned}
$$

The summation on each of the first five lines of the right-hand side of equation (62) is over all pairs of nonnegative integers whose sum does not exceed $\mu$. Therefore, we may apply formulas (55)-(59), $0 \leqq r \leqq \mu$, to show that terms in the first four of these lines vanish pairwise, and that the fifth line vanishes together with the terms in the sixth line for which $s=0$. We are left with

$$
\begin{aligned}
\sum_{\substack{i+j=\mu \\
i, j \geqq 0}} \phi_{i}\left(\phi_{j}\left(x_{1}, x_{2}\right), x_{3}\right)-\phi_{i}\left(x_{1}, \phi_{j}\left(x_{2}, x_{3}\right)\right) \\
=\sum_{r=0}^{\mu} \sum_{\substack{s+q=r \\
s>0}} f_{s}\left(g_{q}\left(a_{1}, a_{2}\right), a_{3}\right)-f_{s}\left(a_{1}, g_{q}\left(a_{2}, a_{3}\right)\right) .
\end{aligned}
$$

In the hypotheses at the beginning of the section no statement was made about any relationship between the extension (i.e., deformation) of $g$ and the law of multiplication $A \otimes M \rightarrow M$, which was made to depend, via the deformation $F_{t}$, upon $t$. That such a connection or compatibility condition is needed is made clear in the present theorem, which will say, when proven, that the right-hand side of (63) vanishes. If $G_{t}$ is to be a coycle, not just in $Z_{\Phi}^{2}(M)$, but with respect to the entire structure, whose multiplication at time $t$ is given by (60), then we must have the compatibility condition

$$
\begin{aligned}
\delta G_{t}\left(a_{1}, a_{2}, a_{3}\right) & =F_{t}\left(a_{1}, G_{t}\left(a_{2}, a_{3}\right)\right)-G_{t}\left(\Phi_{t}\left(a_{1}, a_{2}\right), a_{3}\right) \\
& +G_{t}\left(a_{1}, \Phi_{t}\left(a_{2}, a_{3}\right)\right)-F_{t}\left(G_{t}\left(a_{1}, a_{2}\right), a_{3}\right)=0
\end{aligned}
$$

or, in terms of coefficients of powers of $t$, for all $r \geqq 0$,

$$
\begin{gathered}
\sum_{\substack{p+q=r \\
p, q \geqq 0}}\left(f_{p}\left(a_{1}, g_{q}\left(a_{2}, a_{3}\right)\right)-g_{q}\left(\varphi_{p}\left(a_{1}, a_{2}\right), a_{3}\right)+g_{q}\left(a_{1}, \varphi_{p}\left(a_{2}, a_{3}\right)\right)\right. \\
\left.-f_{p}\left(g_{q}\left(a_{1}, a_{2}\right), a_{3}\right)\right)=0 .
\end{gathered}
$$


The summation over the middle two terms together with the extremes for $p=0$ is zero by (50). Thus the compatibility condition (65) becomes for $r \geqq 0$,

$$
\sum_{\substack{p+q=r \\ p>0}} f_{p}\left(a_{1}, g_{q}\left(a_{2}, a_{3}\right)\right)-f_{p}\left(g_{q}\left(a_{1}, a_{2}\right), a_{3}\right)=0
$$

When $G_{t}$ is such that, for a given $F_{t},(66)$ vanishes, $G_{t}$ is said to be an extension compatible with the deformation $F_{t}$ of the module structure. Comparing (64) and (66) completes the proof of Theorem 4.

Note that the concept of extension $G_{t}$ of $g \in Z^{2}(A, M)$, compatible with a deformation $F_{t}$ of the module structure, also means that $G_{t}$ will be an extension, as in $\S 13$, with respect to the deformation $\Phi_{t}$ of the algebra $A$. While this fact is discussed more fully in the next section, we point out now that it follows from equation (63) in the proof of Theorem 4. If $\Psi_{t}$ is a deformation of $(A+M)_{g}$, then the left-hand side of (63) is zero, as are the first and last (less the terms for $s=0$ ) lines on the right-hand side. The remaining lines on the right-hand side (including the sixth line for $s=0$ with the fifth) are independent of each other, and as their sum is zero, each is zero. The fifth line together with the $s=0$ terms of the sixth expresses the fact that $G_{t}$ is an extension of $g$ with respect to $\Phi_{t}$.

\section{Investigation of deformations of $A+M$}

Reversing the order of considerations in the previous section, we now examine deformations of an extension of $A$ by $M$, and ask when such deformations induce deformation of $A$ and of the module structure of $M$ over $A$, and extensions of $g \in Z^{2}(A, M)$.

It is well known that the equivalence classes of singular extensions of $M$ by $A$ are in one-one correspondence with the second cohomology classes $H^{2}(A, M)$. Let $g$ be a cocycle in $Z^{2}(A, M)$ and consider the corresponding extension $A+M$ of $A$, with multiplication given by

$$
\left(a_{1}, m_{1}\right)\left(a_{2}, m_{2}\right)=\left(a_{1} a_{2}, a_{1} m_{2}+m_{1} a_{2}+g\left(a_{1}, a_{2}\right)\right) .
$$

Denote this algebra by $(A+M)_{g}$ and let $\Psi_{t}\left(x_{1}, x_{2}\right)=\sum_{i \geqq 0} \phi_{i}\left(x_{1}, x_{2}\right) t^{i}$ be a deformation of $(A+M)_{g}$. Therefore, the $\phi_{i}$ satisfy for $r \geqq 0$

$$
\sum_{\substack{p+q=r \\ p, q \geqq 0}} \phi_{p}\left(\phi_{q}\left(x_{1}, x_{2}\right), x_{3}\right)-\phi_{p}\left(x_{1}, \phi_{q}\left(x_{2}, x_{3}\right)\right)=0 .
$$

Because $\Psi_{t}$ is bilinear and $x_{j}=a_{j}+m_{j}, j=1,2$,

$$
\Psi_{t}\left(x_{1}, x_{2}\right)=\Psi_{t}\left(a_{1}, a_{2}\right)+\Psi_{t}\left(a_{1}, m_{2}\right)+\Psi_{t}\left(m_{1}, a_{2}\right)+\Psi_{t}\left(m_{1}, m_{2}\right)
$$

This can be expressed by 


$$
\begin{aligned}
\phi_{i}\left(x_{1}, x_{2}\right)= & \left(\alpha_{i}\left(a_{1}, a_{2}\right)+\beta_{i}\left(a_{1}, m_{2}\right)+\gamma_{i}\left(m_{1}, a_{2}\right)+\varepsilon_{i}\left(m_{1}, m_{2}\right)\right. \\
& \left.\eta_{i}\left(a_{1}, a_{2}\right)+\rho_{i}\left(a_{1}, m_{2}\right)+\sigma_{i}\left(m_{1}, a_{2}\right)+\mu_{i}\left(m_{1}, m_{2}\right)\right)
\end{aligned}
$$

Substituting (68) into (67), we obtain, as the component in $A$, where terms as grouped according to their arguments,

$$
\begin{aligned}
0=\sum_{\substack{p+q=r \\
p, q \geqq 0}}\left[\alpha_{p}\left(\alpha_{q}\left(a_{1}, a_{2}\right), a_{3}\right)-\alpha_{p}\left(a_{1}, \alpha_{q}\left(a_{2}, a_{3}\right)\right)+\gamma_{p}\left(\eta_{q}\left(a_{1}, a_{2}\right), a_{3}\right)\right. \\
\quad-\beta_{p}\left(a_{1}, \eta_{q}\left(a_{2}, a_{3}\right)\right)+\alpha_{p}\left(\beta_{q}\left(a_{1}, m_{2}\right), a_{3}\right)-\alpha_{p}\left(a_{1}, \gamma_{q}\left(m_{2}, a_{3}\right)\right) \\
\quad+\gamma_{p}\left(\rho_{q}\left(a_{1}, m_{2}\right), a_{3}\right)-\beta_{p}\left(a_{1}, \sigma_{q}\left(m_{2}, a_{3}\right)\right)+\alpha_{p}\left(\gamma_{q}\left(m_{1}, a_{2}\right), a_{3}\right) \\
\quad-\gamma_{p}\left(m_{1}, \alpha_{q}\left(a_{2}, a_{3}\right)\right)+\gamma_{p}\left(\sigma_{q}\left(m_{1}, a_{2}\right), a_{3}\right)-\varepsilon_{p}\left(m_{1}, \eta_{q}\left(a_{2}, a_{3}\right)\right) \\
\quad+\alpha_{p}\left(\varepsilon_{q}\left(m_{1}, m_{2}\right), a_{3}\right)-\varepsilon_{p}\left(m_{1}, \sigma_{q}\left(m_{2}, a_{3}\right)\right)+\gamma_{p}\left(\mu_{q}\left(m_{1}, m_{2}\right), a_{3}\right) \\
\quad-\gamma_{p}\left(m_{1}, \gamma_{q}\left(m_{2}, a_{3}\right)\right)+\beta_{p}\left(\alpha_{q}\left(a_{1}, a_{2}\right), m_{3}\right)-\beta_{p}\left(a_{1}, \rho_{q}\left(a_{2}, m_{3}\right)\right) \\
\quad+\varepsilon_{p}\left(\eta_{q}\left(a_{1}, a_{2}\right), m_{3}\right)-\alpha_{p}\left(a_{1}, \beta_{q}\left(a_{2}, m_{3}\right)\right)+\beta_{p}\left(\beta_{q}\left(a_{1}, m_{2}\right), m_{3}\right) \\
\quad-\alpha_{p}\left(a_{1}, \varepsilon_{q}\left(m_{2}, m_{3}\right)\right)+\varepsilon_{p}\left(\rho_{q}\left(a_{1}, m_{2}\right), m_{3}\right)-\beta_{p}\left(a_{1}, \mu_{q}\left(m_{2}, m_{3}\right)\right) \\
\quad+\beta_{p}\left(\gamma_{q}\left(m_{1}, a_{2}\right), m_{3}\right)-\gamma_{p}\left(m_{1}, \beta_{q}\left(a_{2}, m_{3}\right)\right)+\varepsilon_{p}\left(\sigma_{q}\left(m_{1}, a_{2}\right), m_{3}\right) \\
\quad-\gamma_{p}\left(m_{1}, \beta_{q}\left(a_{2}, m_{3}\right)\right)+\beta_{p}\left(\varepsilon_{q}\left(m_{1}, m_{2}\right), m_{3}\right)-\gamma_{p}\left(m_{1}, \varepsilon_{q}\left(m_{2}, m_{3}\right)\right) \\
\left.\quad+\varepsilon_{p}\left(\mu_{q}\left(m_{1}, m_{2}\right), m_{3}\right)-\varepsilon_{p}\left(m_{1}, \mu_{q}\left(m_{2}, m_{3}\right)\right)\right] .
\end{aligned}
$$

Equation (69) and the corresponding equation for the $M$-component of (67) contain much information but little is accessible without further assumptions. For all $r$ the summation over $p, q \geqq 0$ with $p+q=r$ of the terms in each row is zero, as is seen by letting first $m_{1}=m_{2}=m_{3}=0$, then $m_{1}=a_{2}=m_{3}=0$, etc.

Since $\Psi_{t}$ gives a deformation of $(A+M)_{g}$, we have $\beta_{0}=\gamma_{0}=\varepsilon_{0}=$ $\mu_{0}=0$,

and $\alpha_{0}\left(a_{1}, a_{2}\right)=a_{1} a_{2}, \rho_{0}\left(a_{1}, m_{2}\right)=a_{1} m_{2}, \sigma_{0}\left(m_{1}, a_{2}\right)=m_{1} a_{2}$ and $\eta_{0}=g$. Assuming $\beta_{p}=\gamma_{p}=0$ for all $p$, we have from (69)

$$
\begin{aligned}
& \sum_{\substack{p+q=r \\
p, q \geqq 0}} \alpha_{p}\left(\alpha_{q}\left(a_{1}, a_{2}\right), a_{3}\right)-\alpha_{p}\left(a_{1}, \alpha_{q}\left(a_{2}, a_{3}\right)\right)=0, \\
& \sum_{\substack{p+q=r \\
p, q \geqq 0}} \varepsilon_{p}\left(\sigma_{q}\left(m_{1}, a_{2}\right), m_{3}\right)=0
\end{aligned}
$$

and additional relations involving $\alpha, \varepsilon$, and $\sigma ; \alpha, \varepsilon$, and $\rho$; and $\varepsilon$ and $\eta$. The only relation of immediate significance is (70) which says $\sum_{i \geqq 0} \alpha_{i} t^{i}$ is a deformation of $A$ induced by $\Psi_{t}$. Considering (71), and assuming $A$ has a unit, let $a_{2}$ be the unit of $A$. Sine $\varepsilon_{0}=0$ and $\sigma_{0}\left(m_{1}, a_{2}\right)=m_{1}$, (71) implies $\varepsilon_{1}=0$, and recursively, $\varepsilon_{r}=0$ for all $r$. 
Under the assumption that $\beta_{p}=\gamma_{p}=0$ for all $p$, we have from the equation for the $M$-component of (67)

$$
\begin{aligned}
\delta \eta_{t}\left(a_{1}, a_{2}, a_{3}\right)- & \sum_{\substack{p+q=r \\
q>0}} \eta_{p}\left(\alpha_{q}\left(a_{1}, a_{2}\right), a_{3}\right)-\eta_{p}\left(a_{1}, \alpha_{q}\left(a_{2}, a_{3}\right)\right) \\
& =\sum_{\substack{p+q=r \\
q>0}} \sigma_{q}\left(\eta_{p}\left(a_{1}, a_{2}\right), a_{3}\right)-\rho_{q}\left(a_{1}, \eta_{p}\left(a_{2}, a_{3}\right)\right)
\end{aligned}
$$

$$
\sum_{\substack{p+q=r \\ p, q \geqq 0}} \sigma_{p}\left(\rho_{q}\left(a_{1}, m_{2}\right), a_{3}\right)-\rho_{p}\left(a_{1}, \sigma_{q}\left(m_{2}, a_{3}\right)\right)=0
$$

$$
\sum_{\substack{p+q=r \\ p, q \geqq 0}} \mu_{p}\left(m_{1}, \eta_{q}\left(a_{2}, a_{3}\right)\right)=\sum_{\substack{p+q=r \\ p, q \geqq 0}} \sigma_{p}\left(\sigma_{q}\left(m_{1}, a_{2}\right), a_{3}\right)-\sigma_{p}\left(m_{1}, \alpha_{q}\left(a_{2}, a_{3}\right)\right),
$$

$$
\sum_{\substack{p+q=r \\ p, q \geqq 0}} \mu_{p}\left(\eta_{q}\left(a_{1}, a_{2}\right), m_{3}\right)=\sum_{\substack{p+q=r \\ p, q \geqq 0}} \rho_{p}\left(a_{1}, \rho_{q}\left(a_{2}, m_{3}\right)\right)-\rho_{p}\left(\alpha_{q}\left(a_{1}, a_{2}\right), m_{3}\right),
$$

$$
\sum_{\substack{p+q=r \\ p, q \geqq 0}} \mu_{p}\left(\mu_{q}\left(m_{1}, m_{2}\right), m_{3}\right)-\mu_{p}\left(m_{1}, \mu_{q}\left(m_{2}, m_{3}\right)\right)=0
$$

and additional relations involving $\mu$ and $\sigma ; \mu$ and $\rho$; and $\mu, \sigma$, and $\rho$.

Of course $\mu_{0}=0$, but it may be that for some $n, \mu_{n} \neq 0$. Let $n$ be the least such index. Setting $r=2 n$ in (76), we see that $\mu_{n}$ defines an associative multiplication on $M$. Thus it might be the case that $A$ has the structure of a module over the associative algebra $M$, not at $t=0$, but under the deformation $\Psi_{t}$. The assumption that $\varepsilon_{p}=0$, for all $\rho$, suffices to give (76).

Relation (73) corresponds to (58) in the previous section, where we now distinguish between left multiplications $\left(\rho_{0}\right)$ by $A$, and right multiplications $\left(\sigma_{0}\right)$ by $A$. Interpreting $\sum_{i \geqq 0} \rho_{i} t^{i}, \sum_{i \geqq 0} \sigma_{i} t^{i}$ as deformations of the left (respectively right) module structures of $M$ over $A$, we have from (73) that they are compatible. The left-hand side of (72) equals (59), while the right-hand side is the compatibility condition (66). But the fact that $\Psi_{t}$ is a deformation of $(A+M)_{g}$ does not imply these two quantities are independently zero.

The assumption that $\mu_{p}=0$, for all $\rho$, reduces (74) and (75) to (57) and (56), respectively, stating that $\sum_{i \geqq 0} \sigma_{i} t^{i}\left(\sum_{i \geqq 0} \rho_{i} t^{i}\right)$ give deformations of the right (left) module structure of $M$ over $A$, compatible with the deformation $\sum_{i \geqq 0} \alpha_{i} t^{i}$ of $A$. The converse would not seem to be true.

We gather the conclusions of the preceding discussion in

Theorem 5. A deformation $\Psi_{t}=\sum_{i \geq 0} \phi_{i} t^{i}$ of the form (68), of $(A+$ 
$M)_{g}$ for which $\beta_{p}=\gamma_{p}=0$ for all $p$ satisfies the following statements:

i) $\varepsilon_{p}=0$ for all $p$.

ii) $\Psi_{t}$ induces a deformation of $A$.

iii) $\Psi_{t}$ induces an extension of $g$ if and only if such an extension is compatible with the deformed module structure.

iv) If in addition $\mu_{p}=0$ for all $p, \Psi_{t}$ induces a deformation of the twosided module structure of $M$ over $A$ compatible with the induced deformation of $A$.

v) $\Psi_{t}$ induces an associative multiplication on $M$ if the $\mu_{p}$ do not vanish identically.

The development of the module deformation cohomology and discussion of an exact cohomology diagram (cf. (24)) associated to the deformation cohomology of the central term of the exact sequence,

$$
0 \rightarrow M \rightarrow(A+M)_{g} \rightarrow A \rightarrow 0
$$

for given $g \in Z^{2}(A, M)$ is left to a later paper. The strong parallel between this exact sequence and a similar sequence in the deformation theory of complex analytic fiber bundles will be discussed in that paper.

\section{Bibliography}

[1] C. Chevalley \& S. Eilenberg, Cohomology theory of Lie groups and Lie algebras, Trans. Amer. Math. Soc. 63 (1948) 85-124.

[2] A. Frölicher \& A. Nijenhuis, A theorem on stability of complex structures, Proc. Nat. Acad. Sci. U.S.A. 43 (1957) 239-241.

[3] M. Gerstenhaber, The cohomology structure of an associative ring, Ann. of Math. 78 (1963) 267-289.

[4] - A uniform cohomology theory for algebras, Proc. Nat. Acad. Sci. U.S.A. 51 (1964) 626-629.

[5] — On the deformation of rings and algebras, Ann. of Math. 79 (1964) 59-104.

[6] - On the deformation of rings and algebras II, Ann. of Math. 84 (1966) 1-19.

[7] — On the deformation of rings and algebras III. Obstructions to derivations and construction of division algebras, to appear.

[8] P. A. Griffiths, The extension problem for compact submanifolds of complex manifolds I (the case of a trivial normal bundle), Proc. Conf. Complex Analysis (Minneapolis), Springer, Berlin, 1965, 113-142.

[9] V. W. Guillemin \& S. Sternberg, Deformation theory of pseudogroup structures, Harvard Report (polycopied), 1964, to be published in Ann. of Math. 
[10] A. Haefliger, Structures feuilletées et cohomologie à valuer dans un faisceau de groupoides, Comment. Math. Helv. 32 (1958) 248-329.

[11] D. Harrison, Commutative algebras and cohomology, Trans. Amer. Math. Soc. 104 (1962) 191-204.

[12] G. Hochschild, On the cohomology groups of an associative algebra, Ann. of Math. 46 (1945) 58-67.

[13] K. Kodaira, L. Nirenberg, \& D. C. Spencer, On the existence of deformations of complex analytic structures, Ann. of Math. 68 (1958) $450-459$.

[14] K. Kodaira \& D. C. Spencer, A theorem of completeness for complex analytic fiber spaces, Acta Math. 100 (1958) 281-294.

[15] - On deformations of complex analytic structures I-II, Ann. of Math. 67 (1958) 328-466.

[16] M. Kuranishi, On the Locally complete families of analytic structures, Ann. of Math. 75 (1962) 536-577.

[17] — New proof for the existence of locally complete families of complex structures, Proc. Conf. Complex Analysis (Minneapolis), Springer, Berlin, 1965, 142-154.

[18] A. Nijenhuis \& R. W. Richardson, Jr., Cohomology and deformations of algebraic structures, Bull. Amer. Math. Soc. 70 (1964) 406-411.

[19] — Cohomology and deformations in graded Lie algebras, Bull. Amer. Math. Soc. 72 (1966) 1-29.

[20] D. S. Rim, Deformation of transitive Lie algebras, Ann. of Math. 83 (1966) 339-357.

[21] D. C. Spencer, Some remarks on homological analysis and structures, Proc. Symposia in Pure Math., Amer. Math. Soc. 3 (1961) $56-86$.

[22] - Deformation of structures on manifolds defined by transitive, continuous pseudogroups I-II, Ann. of Math. 76 (1962) 306-445.

[23] —, Deformation of structures on manifolds defined by transitive, continuous pseudogroups. Part III: Structures defined by elliptic pseudogroups, Ann. of Math. 81 (1965) 389-450.

[24] A. Weil, Foundations of algebraic geometry, Colloq. Publ. vol. 29, revised edition, Amer. Math. Soc., 1962.

[25] O. Zariski \& P. Samuel, Commutative algebra, vol. II, Van Nostrand, Princeton, 1960. 